\title{
Western Moyamoya Phenotype: A Scoping Review
}

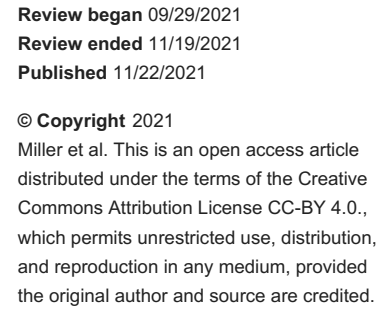

\author{
Raphael Miller ${ }^{1}$, Santiago R. Unda ${ }^{1}$, Ryan Holland ${ }^{1}$, David J. Altschul ${ }^{1}$ \\ 1. Neurological Surgery, Montefiore/Albert Einstein College of Medicine, Bronx, USA \\ Corresponding author: Raphael Miller, raphael.miller@einsteinmed.org
}

\begin{abstract}
Moyamoya, a rare angiographic finding, is characterized by chronic and progressive stenosis at the terminal end of the internal carotid artery, followed by collateralization of the cerebral vasculature at the base of the skull. Coined by Suzuki and Takaku in 1969, the term "moyamoya" means a "puff of smoke" in Japanese, a reference to the angiographic appearance of moyamoya collateralization. Moyamoya is most commonly found in East Asian countries, where much governmental and civilian effort has been expended to characterize this unique disease process. However, despite its rarity, the occurrence of moyamoya in Western countries is associated with significant divergence regarding incidence, gender, sex, age at diagnosis, clinical presentation, and outcomes. Here, we attempted to review the Western literature on moyamoya presentation using the PubMed database to characterize the Western phenotype of moyamoya. We were guided by the Preferred Reporting Items for Systematic Reviews and Meta-Analyses extension for scoping reviews (PRISMA-ScR). We reviewed papers generated from a search with keywords "moyamoya case report," those reported from a Western institution, and those reported on a relevant association. Our scoping review demonstrated various clinical associations with moyamoya. Moreover, we summarized the demographic profile and clinical symptomatology, as well as reported disease associations to better elucidate the Western phenotype of moyamoya.
\end{abstract}

Categories: Neurology, Radiology, Neurosurgery

Keywords: systematic review, moyamoya disease (mmd), moyamoya angiopathy, article review, clinical phenotype

\section{Introduction And Background}

Moyamoya, first described as a distinct disease entity in Japan in 1957, is a chronic and progressive stenosis at the supraclinoid, terminal end of the internal carotid arteries and their proximal branches, and the proximal portions of the middle and anterior cerebral arteries, which can have a unilateral or bilateral presentation. Stenosis of cerebral vasculature leads to the collateralization of the lenticulostriate, leptomeningeal, cortical surface, or Dural vasculature at the base of the skull. A characteristic angiographic finding follows, with a distinct vascular blush on angiography, described by Suzuki and Takaku in 1969 as a "puff of smoke" [1] or "moyamoya" in Japanese. Because of its irreversible and progressive course, Suzuki suggested a time-based classification system that reflects the extent of intracranial artery involvement [1], which is still in use today and is referred to, eponymously, as the Suzuki scale [2].

Moyamoya was originally thought to arise spontaneously and not as a result of a congenital malformation. Indeed, Suzuki wrote about reporting "repeatedly that the disease is an acquired one in its origin resulting from a stricture at the carotid fork due to unknown causes [3]." More recently, in 2011, the ring finger protein 213 (RNF213) gene was identified using genome-wide association studies as the first discovered susceptibility gene for moyamoya [4]. While the exact pathogenic role of RNF213 in moyamoya is unknown, it has been shown to play a role in angiogenesis [5] and endothelial inflammatory processes [6]. Subsequently, the p.R4810K variant of RNF213 was identified as a common gene variant, present in $90 \%$ of Japanese moyamoya patients, $79 \%$ of Korean moyamoya patients, and $23 \%$ of Chinese moyamoya patients $[7,8]$. Other variants of lesser prevalence have also been identified, including the R179 and R258 mutations to the actin alpha 2 (ACTA2) gene [9]. However, the unknown etiology of the remainder, as well as the fact that the p.R4810K RNF213 variant has not been found in Caucasian moyamoya patients, suggests etiologic heterogeneity for moyamoya vasculopathy [7].

Patients are said to have moyamoya syndrome (MMS) instead of moyamoya disease (MMD) when it occurs together with a well-recognized associated condition, including, but not limited to, sickle cell disease, Down's syndrome, neurofibromatosis 1, cranial irradiation, type 1 diabetes mellitus, and Graves' disease $[10,11]$. MMS is alternatively known as secondary moyamoya or quasi-moyamoya and can be unilateral or bilateral, whereas MMD is alternatively known as primary moyamoya and is, by definition, bilateral [10]. Well-recognized conditions associated with moyamoya are growing in number as research into this strange and mysterious phenotype advances. In this paper, we aim to collate all conditions associated with moyamoya from reports in the Western literature using the PubMed database to elucidate the full spectrum and associations of the Western moyamoya phenotype. Therefore, we chose to avoid the use of these diagnostic descriptors altogether (i.e., primary, secondary, etc.) as the distinction between them is expected to blur as the category of "well-recognized associated conditions" expands. As a secondary goal, we planned to apply similar methodologies to collate and elucidate the symptoms normally associated with 


\section{Cureus}

moyamoya for greater appreciation of this wide-ranging condition.

MMD and MMS occur mostly in East Asian populations where much governmental and civilian effort has been expended to characterize this unique disease process, which has highlighted important features of the moyamoya phenotype in a broad and robust collection of literature. However, the rarity with which MMS afflicts the Western world presents a unique challenge in the study of its Western phenotype, known to present as a distinct clinical entity [12]. Moreover, various studies in the United States have found rates of MMD increasing over time [13,14], making characterization of associated conditions especially critical. Table 1 references several national in-patient studies reported in the last several decades that are representative of roughly $20 \%$ of all US hospital admissions for those years; it demonstrates rising moyamoya rates.

\begin{tabular}{|c|c|c|c|c|c|}
\hline Study & Reference & Male & Female & $\mathbf{N}$ & Inclusion criteria \\
\hline Moyamoya and inflammation & [15] & $30 \%$ & $70 \%$ & $2,633^{*}$ & 2009-2012 \\
\hline $\begin{array}{l}\text { Prevalence and characteristics of concurrent Down } \\
\text { syndrome in patients with moyamoya disease }\end{array}$ & [16] & $29.5 \%$ & $70.5 \%$ & 13,275 & $\begin{array}{l}2002-2009 \text { NIS study of } \\
\text { moyamoya patients with Down } \\
\text { Syndrome }\end{array}$ \\
\hline Moyamoya disorder in the United States & [12] & $28 \%$ & $72 \%$ & 11,163 & $\begin{array}{l}\text { 2002-2008 NIS study of } \\
\text { moyamoya patients }\end{array}$ \\
\hline $\begin{array}{l}\text { Epidemiological and clinical features of patients with } \\
\text { moyamoya disease in the USA) }\end{array}$ & [14] & $29.5 \%$ & $70.5 \%$ & 7,473 & $\begin{array}{l}\text { 2005-2008 NIS study of } \\
\text { moyamoya patients }\end{array}$ \\
\hline Characterization of inpatient moyamoya & [17] & $38.1 \%$ & $61.9 \%$ & $2,247^{*}$ & $\begin{array}{l}\text { 1988-2004 NIS study of } \\
\text { moyamoya patients }\end{array}$ \\
\hline $\begin{array}{l}\text { Treatment course and outcomes after revascularization } \\
\text { surgery for moyamoya disease in adults }\end{array}$ & [13] & $25.4 \%$ & $74.6 \%$ & 201 & $\begin{array}{l}2013 \text { NRD study of moyamoya } \\
\text { patients that underwent } \\
\text { revascularization surgery }\end{array}$ \\
\hline $\begin{array}{l}\text { Socioeconomic and demographic disparities of moyamoya } \\
\text { disease in the United States }\end{array}$ & [18] & $31.7 \%$ & $68.3 \%$ & $\begin{array}{l}\text { Not } \\
\text { available }\end{array}$ & $\begin{array}{l}2008-2015 \text { NIS study of } \\
\text { moyamoya patients }\end{array}$ \\
\hline
\end{tabular}

TABLE 1: Relative moyamoya count and male to female ratios in four separate studies.

NIS: National Inpatient Sample; NRD: Nationwide Readmissions Database

To our knowledge, this is the first attempt to date to collect all known case reports in the literature of Western-type moyamoya for a comprehensive analysis of the unique features with which Western moyamoya can be encountered. Guided by the Preferred Reporting Items for Systematic Reviews and MetaAnalyses extension for scoping reviews (PRISMA-ScR) (Appendices, Figures 2, 3), we aim to answer the research question: what is known about the demographic profiles and clinical symptomatology of Western moyamoya and the diseases that are associated with it?

\section{Review}

\section{Methodology}

Protocol and Registration

Our scoping review protocol was not registered.

Eligibility Criteria

We included English-language papers identified by their titles as etiology of moyamoya or moyamoya symptoms. We excluded papers for which the first author's address was located in Asia (if the first author's address was unavailable, then the first available address of any author thereafter was used).

Information Sources and Search

A literature search of PubMed was performed to collate case reports on moyamoya. Duplicates were removed manually by the authors. The following search term was used to screen for relevant papers on PubMed published in the preceding 10 years on August 9, 2020: “moyamoya case report." 


\section{Cureus}

Selection of Sources of Evidence

Papers were initially screened by their titles only. However, to better define their status according to the inclusion criteria, the abstract and/or paper were read to clarify ambiguities. Further, for the purpose of the review, relevant papers were read and mined for relevant content. Finally, the association between moyamoya and each symptom and associated pathology was explored in relevant additional literature.

Data Charting Process and Data Items

Data were manually extracted as profiles of patients with major findings relevant to the review and were added to an excel spreadsheet. Unique findings were further reviewed outside of the search criteria for the Discussion section.

Synthesis of Results

Results were grouped based on the association reported by the paper. Additionally, they were classified by the country of the first author's address according to the inclusion/exclusion criteria.

\section{Results}

Primary search on PubMed using keywords "moyamoya case report” for primary sources, filtered to only include papers published in the last 10 years, yielded 725 papers. A total of 368 papers were removed on applying exclusion criteria, leaving 357 papers. From these papers, 153 were selected for further analysis using our inclusion criteria. See Figure 1 for a flowchart schematic of literature search results.

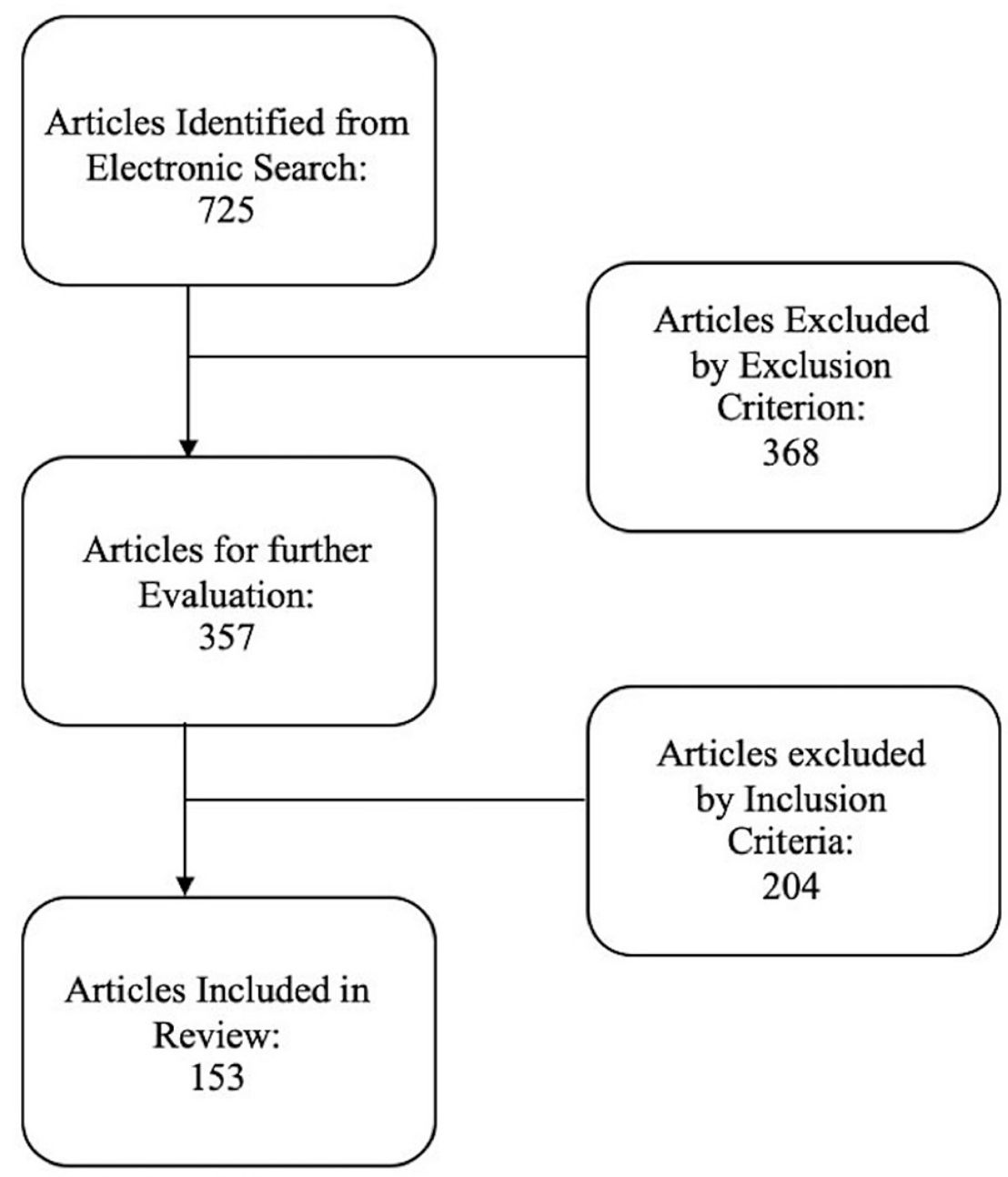

FIGURE 1: A flowchart schematic of papers identified by the search criteria.

Table 2 presents a full list of all papers identified by our search criteria. 


\section{Cureus}

\begin{tabular}{|c|c|c|c|c|c|}
\hline Number & Reference & PMID & Title & Category & Association \\
\hline 1 & [19] & 26795912 & $\begin{array}{l}\text { Transient intraoperative central diabetes insipidus in } \\
\text { moyamoya patients undergoing revascularization surgery: } \\
\text { a mere coincidence? }\end{array}$ & Renal & Diabetes insipidus \\
\hline 2 & [20] & 23537685 & $\begin{array}{l}\text { What lies beneath: Fabry nephropathy in a female patient } \\
\text { with severe cerebrovascular disease }\end{array}$ & Renal & Fabry nephropathy \\
\hline 3 & [21] & 29351941 & $\begin{array}{l}\text { Moyamoya tipping point: fatal bilateral MCA territory } \\
\text { infarction following cocaine abuse }\end{array}$ & Drugs & Cocaine abuse \\
\hline 4 & [22] & 20823033 & $\begin{array}{l}\text { Association of rapidly progressive moyamoya syndrome } \\
\text { with bevacizumab treatment for glioblastoma in a child } \\
\text { with neurofibromatosis type } 1\end{array}$ & Drugs & $\begin{array}{l}\text { Bevacizumab for } \\
\text { glioblastoma }\end{array}$ \\
\hline 5 & [23] & 30861282 & $\begin{array}{l}\text { Tyrosine kinase inhibitor toxicity manifesting as comorbid } \\
\text { Moyamoya syndrome and obstructive coronary artery } \\
\text { disease: a case report and review of the literature }\end{array}$ & Drugs & $\begin{array}{l}\text { Tyrosine kinase } \\
\text { inhibitor toxicity }\end{array}$ \\
\hline 6 & [24] & 31658349 & $\begin{array}{l}\text { Neurofibromatosis type } 1 \text { associated with moyamoya } \\
\text { syndrome. Case report and review of the literature }\end{array}$ & Neurocutaneous & $\begin{array}{l}\text { Neurofibromatosis } \\
\text { type } 1\end{array}$ \\
\hline 7 & [25] & 24085541 & $\begin{array}{l}\text { Moyamoya syndrome related to neurofibromatosis of type } \\
\text { 1: a case report }\end{array}$ & Neurocutaneous & $\begin{array}{l}\text { Neurofibromatosis } \\
\text { type } 1\end{array}$ \\
\hline 8 & [26] & 28279525 & $\begin{array}{l}\text { "Ivy sign" and moyamoya disease in a child with } \\
\text { neurofibromatosis type } 1\end{array}$ & Neurocutaneous & $\begin{array}{l}\text { Neurofibromatosis } \\
\text { type } 1\end{array}$ \\
\hline 9 & [27] & 24952383 & Moyamoya syndrome and neurofibromatosis type 1 & Neurocutaneous & $\begin{array}{l}\text { Neurofibromatosis } \\
\text { type } 1\end{array}$ \\
\hline 10 & [28] & 28620564 & $\begin{array}{l}\text { Moyamoya syndrome in a child with neurofibromatosis } \\
\text { type 1: magnetic resonance imaging as a tool for clinical } \\
\text { decision making }\end{array}$ & Neurocutaneous & $\begin{array}{l}\text { Neurofibromatosis } \\
\text { type } 1\end{array}$ \\
\hline 11 & [29] & 29364453 & $\begin{array}{l}\text { Moyamoya syndrome associated with neurofibromatosis } \\
\text { type } 1 \text { in a pediatric patient }\end{array}$ & Neurocutaneous & $\begin{array}{l}\text { Neurofibromatosis } \\
\text { type } 1\end{array}$ \\
\hline 12 & [30] & 24801636 & $\begin{array}{l}\text { Adult-onset moyamoya disease in a patient with } \\
\text { neurofibromatosis type } 1\end{array}$ & Neurocutaneous & $\begin{array}{l}\text { Neurofibromatosis } \\
\text { type } 1\end{array}$ \\
\hline 13 & [31] & 23661665 & $\begin{array}{l}\text { Simultaneous moyamoya disease and cervical spinal } \\
\text { cord low-grade astrocytoma in a child with } \\
\text { neurofibromatosis type } 1\end{array}$ & Neurocutaneous & $\begin{array}{l}\text { Neurofibromatosis } \\
\text { type } 1\end{array}$ \\
\hline 14 & [32] & 21603789 & $\begin{array}{l}\text { Moyamoya syndrome associated with neurofibromatosis } \\
\text { type I in a pediatric patient }\end{array}$ & Neurocutaneous & $\begin{array}{l}\text { Neurofibromatosis } \\
\text { type } 1\end{array}$ \\
\hline 15 & [33] & 21271658 & $\begin{array}{l}\text { Cerebrovasculopathy in NF1 associated with ocular and } \\
\text { scalp defects }\end{array}$ & Neurocutaneous & $\begin{array}{l}\text { Neurofibromatosis } \\
\text { type } 1\end{array}$ \\
\hline 16 & [34] & 24141273 & $\begin{array}{l}\text { Poor wound healing after pial synangiosis in } 2 \text { children } \\
\text { with moyamoya vasculopathy associated with } \\
\text { neurofibromatosis type } 1\end{array}$ & Neurocutaneous & $\begin{array}{l}\text { Neurofibromatosis } \\
\text { type } 1\end{array}$ \\
\hline 17 & [35] & 28237043 & $\begin{array}{l}\text { Tuberous sclerosis complex and polycystic kidney } \\
\text { disease contiguous gene syndrome with moyamoya } \\
\text { disease }\end{array}$ & Neurocutaneous & $\begin{array}{l}\text { Tuberous sclerosis } \\
\text { complex }\end{array}$ \\
\hline 18 & [36] & 24878482 & Moyamoya syndrome associated with mucolipidosis-II & Metabolic & Mucolipidosis 2 \\
\hline 19 & [37] & 25207193 & $\begin{array}{l}\text { Protein } \mathrm{S} \text { deficiency and an adult case with moyamoya } \\
\text { syndrome that presented with primary intraventricular } \\
\text { haemorrhage }\end{array}$ & Metabolic & Protein S deficiency \\
\hline 20 & [38] & 29843966 & $\begin{array}{l}\text { CD59 deficiency presenting as polyneuropathy and } \\
\text { moyamoya syndrome with endothelial abnormalities of } \\
\text { small brain vessels }\end{array}$ & Metabolic & CD59 deficiency \\
\hline 21 & [39] & 30021915 & $\begin{array}{l}\text { Severe hyperhomocysteinemia manifesting as } \\
\text { moyamoya vasculopathy and Henoch-Schonlein purpura }\end{array}$ & Metabolic & Hyperhomocyst \\
\hline
\end{tabular}




\section{Cureus}

\begin{tabular}{|c|c|c|c|c|c|}
\hline 22 & [40] & 27321952 & $\begin{array}{l}\text { Unusual association between lysinuric protein intolerance } \\
\text { and moyamoya vasculopathy }\end{array}$ & Metabolic & $\begin{array}{l}\text { Lysinuric protein } \\
\text { intolerance }\end{array}$ \\
\hline 23 & [41] & 22034792 & $\begin{array}{l}\text { Moyamoya syndrome with arteriovenous dural fistula } \\
\text { after head trauma }\end{array}$ & Physical trauma & Head trauma \\
\hline 24 & [42] & 21830458 & $\begin{array}{l}\text { Moyamoya disease in a patient with brain tumor: case } \\
\text { report }\end{array}$ & Physical trauma & Brain tumor \\
\hline 25 & [43] & 28698088 & $\begin{array}{l}\text { Microsurgical repair of ruptured aneurysms associated } \\
\text { with moyamoya-pattern collateral vessels of the middle } \\
\text { cerebral artery: a report of two cases }\end{array}$ & Physical trauma & $\begin{array}{l}\text { Postsurgical repair of } \\
\text { a ruptured aneurysm }\end{array}$ \\
\hline 26 & [44] & 27857839 & $\begin{array}{l}\text { Fatal outcome in a Hispanic woman with moyamoya } \\
\text { syndrome and Graves' disease }\end{array}$ & Autoimmune & Graves' disease \\
\hline 27 & [45] & 21863249 & $\begin{array}{l}\text { Dual anca positivity in a child with moyamoya-like } \\
\text { cerebral vascular changes: an unusual presentation with } \\
\text { sudden homonymous hemianopsia }\end{array}$ & Autoimmune & $\begin{array}{l}\text { Dual antineutrophil } \\
\text { cytoplasmic } \\
\text { antibodies positivity }\end{array}$ \\
\hline 28 & [46] & 30017593 & $\begin{array}{l}\text { Moyamoya vasculopathy with anti-SCL-70 antibodies: a } \\
\text { case report and review of the literature }\end{array}$ & Autoimmune & $\begin{array}{l}\text { Anti-SCL-70 } \\
\text { antibodies }\end{array}$ \\
\hline 29 & [47] & 30732990 & $\begin{array}{l}\text { Reply to 'Moyamoya vasculopathy with anti-SCL-70 } \\
\text { antibodies: A case report and review of the literature' }\end{array}$ & Autoimmune & $\begin{array}{l}\text { Anti-SCL-70 } \\
\text { antibodies }\end{array}$ \\
\hline 30 & [48] & 23651859 & $\begin{array}{l}\text { Systemic lupus erythematosus due to } \mathrm{C} 1 \mathrm{q} \text { deficiency with } \\
\text { progressive encephalopathy, intracranial calcification and } \\
\text { acquired moyamoya cerebral vasculopathy }\end{array}$ & Autoimmune & $\begin{array}{l}\text { SLE secondary to } \\
\text { C1q deficiency }\end{array}$ \\
\hline 31 & [49] & 30094527 & $\begin{array}{l}\text { Moyamoya complicated by thrombotic cerebrovascular } \\
\text { accident in a Caucasian woman with collagenous colitis }\end{array}$ & Autoimmune & Collagenous colitis \\
\hline 32 & [50] & 27606722 & $\begin{array}{l}\text { Moyamoya disease and systemic sclerosis (MoSys } \\
\text { syndrome): a combination of two rare entities }\end{array}$ & Autoimmune & Systemic sclerosis \\
\hline 33 & [51] & 23380559 & $\begin{array}{l}\text { Concurrent Takayasu arteritis with common variable } \\
\text { immunodeficiency and moyamoya disease }\end{array}$ & Autoimmune & Takayasu arteritis \\
\hline 34 & [52] & 27592246 & $\begin{array}{l}\text { Moyamoya syndrome causing stroke in young women } \\
\text { with type } 1 \text { diabetes }\end{array}$ & Autoimmune & $\begin{array}{l}\text { Diabetes mellitus type } \\
1\end{array}$ \\
\hline 35 & [53] & 21130668 & $\begin{array}{l}\text { Moyamoya syndrome associated with Graves disease: a } \\
\text { case report and review of the literature }\end{array}$ & Autoimmune & Graves' disease \\
\hline 36 & [54] & 29475609 & $\begin{array}{l}\text { Moya-moya syndrome after cranial radiation for optic } \\
\text { glioma with NF1. Case report and literature review of } \\
\text { syndromic cases }\end{array}$ & Radiation & $\begin{array}{l}\text { Cranial radiation for } \\
\text { optic glioma }\end{array}$ \\
\hline 37 & [55] & 27193755 & $\begin{array}{l}\text { Radiation-induced moyamoya syndrome after proton } \\
\text { beam therapy in the pediatric patient: a case series }\end{array}$ & Radiation & Proton beam therapy \\
\hline 38 & [56] & 21783367 & $\begin{array}{l}\text { Radiation-induced moyamoya disease after childhood } \\
\text { astrocytoma }\end{array}$ & Radiation & $\begin{array}{l}\text { Radiation for } \\
\text { childhood } \\
\text { astrocytoma }\end{array}$ \\
\hline 39 & [57] & 24501091 & $\begin{array}{l}\text { The development of moyamoya syndrome after proton } \\
\text { beam therapy }\end{array}$ & Radiation & Proton beam therapy \\
\hline 40 & [58] & 30583132 & $\begin{array}{l}\text { Radiation-induced moyamoya syndrome after proton } \\
\text { therapy in child with clival chordoma: natural history and } \\
\text { surgical treatment }\end{array}$ & Radiation & $\begin{array}{l}\text { Proton beam therapy } \\
\text { for clival chordoma }\end{array}$ \\
\hline 41 & [59] & 31893204 & $\begin{array}{l}\text { Moyamoya syndrome after radiation therapy: a clinical } \\
\text { report }\end{array}$ & Radiation & Radiation therapy \\
\hline 42 & [60] & 28343148 & $\begin{array}{l}\text { De novo mutations in CBL causing early-onset paediatric } \\
\text { moyamoya angiopathy }\end{array}$ & Congenital & CBL mutations \\
\hline 43 & [61] & 26277359 & $\begin{array}{l}\text { Familial moyamoya disease in two Turkish siblings with } \\
\text { same polymorphism in RNF213 gene but different clinical } \\
\text { features }\end{array}$ & Congenital & $\begin{array}{l}\text { RNF213 } \\
\text { polymorphism }\end{array}$ \\
\hline
\end{tabular}




\section{Cureus}

\begin{tabular}{|c|c|c|c|c|c|}
\hline 44 & [62] & 30463371 & $\begin{array}{l}\text { Microduplication of } 15 q 13.3 \text { and microdeletion of } \\
18 q 21.32 \text { in a patient with moyamoya syndrome }\end{array}$ & Congenital & $\begin{array}{l}\text { Microduplication of } \\
15 q 13.3 \text { and } \\
\text { microdeletion of } \\
18 q 21.32\end{array}$ \\
\hline 45 & [63] & 29263223 & $\begin{array}{l}\text { Moyamoya-like cerebrovascular disease in a child with a } \\
\text { novel mutation in myosin heavy chain } 11\end{array}$ & Congenital & $\begin{array}{l}\text { Myosin heavy chain } \\
11 \text { mutation }\end{array}$ \\
\hline 46 & [64] & 27236536 & $\begin{array}{l}\text { Moyamoya syndrome and } 6 \mathrm{p} \text { chromosome } \\
\text { rearrangements: expanding evidences of a new } \\
\text { association }\end{array}$ & Congenital & $\begin{array}{l}6 \mathrm{p} \text { chromosome } \\
\text { rearrangements }\end{array}$ \\
\hline 47 & [65] & 25413039 & $\begin{array}{l}\text { Cerebral cavernous malformations and unilateral } \\
\text { moyamoya in a patient with a new mutation in the KRIT- } \\
1 / \text { CCM1 gene }\end{array}$ & Congenital & $\begin{array}{l}\mathrm{KRIT1} / \mathrm{CCM} 1 \\
\text { mutation }\end{array}$ \\
\hline 48 & [66] & 26777256 & $\begin{array}{l}\text { Disrupted nitric oxide signaling due to GUCY1A3 } \\
\text { mutations increases risk for moyamoya disease, } \\
\text { achalasia and hypertension }\end{array}$ & Congenital & GUCY1A3 mutation \\
\hline 49 & [67] & 28686325 & $\begin{array}{l}\text { RNF213 variants in a child with PHACE syndrome and } \\
\text { moyamoya vasculopathy }\end{array}$ & Congenital & $\begin{array}{l}\text { RNF213 variant, } \\
\text { PHACE syndrome }\end{array}$ \\
\hline 50 & [68] & 26198278 & $\begin{array}{l}\text { Atypical presentation of moyamoya disease in an infant } \\
\text { with a de novo RNF213 variant }\end{array}$ & Congenital & RNF213 variant \\
\hline 51 & [69] & 27611897 & $\begin{array}{l}\text { Surgical outcomes of Majewski osteodysplastic primordial } \\
\text { dwarfism type II with intracranial vascular anomalies }\end{array}$ & Congenital & $\begin{array}{l}\text { Majewski } \\
\text { osteodysplastic } \\
\text { primordial dwarfism } \\
\text { type II }\end{array}$ \\
\hline 52 & [70] & 22527565 & $\begin{array}{l}\text { Medical management of moyamoya disease and } \\
\text { recurrent stroke in an infant with Majewski } \\
\text { osteodysplastic primordial dwarfism type II (MOPD II) }\end{array}$ & Congenital & $\begin{array}{l}\text { Majewski } \\
\text { osteodysplastic } \\
\text { primordial dwarfism } \\
\text { type II }\end{array}$ \\
\hline 53 & [71] & 23337351 & $\begin{array}{l}\text { "Ocular moyamoya" syndrome in a patient with features } \\
\text { of microcephalic osteodysplastic primordial dwarfism type } \\
\text { ॥ }\end{array}$ & Congenital & $\begin{array}{l}\text { Majewski } \\
\text { osteodysplastic } \\
\text { primordial dwarfism } \\
\text { type II }\end{array}$ \\
\hline 54 & [72] & 28926972 & $\begin{array}{l}\text { Juvenile moyamoya and craniosynostosis in a child with } \\
\text { deletion 1p32p31: expanding the clinical spectrum of } \\
\text { 1p32p31 deletion syndrome and a review of the literature }\end{array}$ & Congenital & 1p32p31 deletion \\
\hline 55 & [73] & 21147392 & $\begin{array}{l}\text { Moyamoya disease associated with hereditary } \\
\text { spherocytosis }\end{array}$ & Hematological & $\begin{array}{l}\text { Hereditary } \\
\text { spherocytosis }\end{array}$ \\
\hline 56 & {$[74]$} & 28612582 & $\begin{array}{l}\text { Management in rare association of moyamoya syndrome } \\
\text { and hereditary spherocytosis }\end{array}$ & Hematological & $\begin{array}{l}\text { Hereditary } \\
\text { spherocytosis }\end{array}$ \\
\hline 57 & [75] & 28221268 & $\begin{array}{l}\text { Moyamoya syndrome associated with hereditary } \\
\text { spherocytosis: an emerging clinical entity }\end{array}$ & Hematological & $\begin{array}{l}\text { Hereditary } \\
\text { spherocytosis }\end{array}$ \\
\hline 58 & [76] & 22606383 & $\begin{array}{l}\text { Laparoscopic splenectomy in a child with moyamoya } \\
\text { syndrome, hereditary spherocytosis, and interstitial lung } \\
\text { disease: a mere coincidence or partnership based on } \\
\text { genetic similarities }\end{array}$ & Hematological & $\begin{array}{l}\text { Hereditary } \\
\text { spherocytosis }\end{array}$ \\
\hline 59 & [77] & 21728723 & $\begin{array}{l}\text { Cerebral infarction in the setting of moyamoya in a } \\
\text { pediatric patient with sickle } \beta+\text {-thalassemia }\end{array}$ & Hematological & Sickle $\beta+$-thalassemia \\
\hline 60 & [78] & 25719591 & $\begin{array}{l}\text { Fanconi anemia associated with moyamoya disease in } \\
\text { Saudi Arabia }\end{array}$ & Hematological & Fanconi anemia \\
\hline 61 & [79] & 25178886 & $\begin{array}{l}\text { Moyamoya syndrome in sickle cell anaemia: a cause of } \\
\text { recurrent stroke }\end{array}$ & Hematological & Sickle cell anemia \\
\hline 62 & [80] & 24948625 & $\begin{array}{l}\text { Novel severe hemophilia A and moyamoya (SHAM) } \\
\text { syndrome caused by Xq28 deletions encompassing F8 } \\
\text { and BRCC3 genes }\end{array}$ & Hematological & Hemophilia A \\
\hline
\end{tabular}




\section{Cureus}

\begin{tabular}{|c|c|c|c|c|c|}
\hline 63 & [81] & 25342087 & $\begin{array}{l}\text { Moyamoya syndrome associated with hemoglobin } \\
\text { Southampton (Casper) }\end{array}$ & Hematological & $\begin{array}{l}\text { Hemoglobin } \\
\text { Southampton }\end{array}$ \\
\hline 64 & [82] & 23735661 & $\begin{array}{l}\text { A rare case of moyamoya syndrome in a } \beta \text {-thalassemia } \\
\text { major patient }\end{array}$ & Hematological & $\begin{array}{l}\beta \text {-thalassemia major } \\
\text { patient }\end{array}$ \\
\hline 65 & [83] & 31100171 & $\begin{array}{l}\text { CRAO in moyamoya syndrome associated with } \\
\text { Southampton hemoglobinopathy }\end{array}$ & Hematological & $\begin{array}{l}\text { Hemoglobin } \\
\text { Southampton }\end{array}$ \\
\hline 66 & [84] & 26422091 & $\begin{array}{l}\text { X-linked moyamoya syndrome associated with severe } \\
\text { haemophilia A }\end{array}$ & Hematological & Hemophilia A \\
\hline 67 & [85] & 23953970 & $\begin{array}{l}\text { Moyamoya syndrome associated with sickle cell trait in a } \\
\text { child }\end{array}$ & Hematological & Sickle cell trait \\
\hline 68 & {$[86]$} & 23369159 & Moyamoya syndrome with sickle cell trait & Hematological & Sickle cell trait \\
\hline 69 & [87] & 24571831 & $\begin{array}{l}\text { Ivy sign in mildly symptomatic } \beta \text {-thalassemia intermedia, } \\
\text { with development of moyamoya disease }\end{array}$ & Hematological & $\begin{array}{l}\beta \text {-thalassemia } \\
\text { intermedia }\end{array}$ \\
\hline 70 & [88] & 27843469 & $\begin{array}{l}\text { Moyamoya syndrome associated with Henoch-Schönlein } \\
\text { purpura }\end{array}$ & Hematological & $\begin{array}{l}\text { Henoch-Schönlein } \\
\text { purpura }\end{array}$ \\
\hline 71 & [89] & 21337683 & $\begin{array}{l}\text { Oculoectodermal syndrome with coarctation of the aorta } \\
\text { and moyamoya disease: expanding the phenotype to } \\
\text { include vascular anomalies }\end{array}$ & Syndrome & $\begin{array}{l}\text { Oculoectodermal } \\
\text { syndrome }\end{array}$ \\
\hline 72 & [90] & 28647711 & $\begin{array}{l}\text { Down syndrome and moyamoya disease: unusual cause } \\
\text { of stroke }\end{array}$ & Syndrome & Down syndrome \\
\hline 73 & [91] & 26778511 & $\begin{array}{l}\text { Management of moyamoya syndrome in patients with } \\
\text { Noonan syndrome }\end{array}$ & Syndrome & Noonan syndrome \\
\hline 74 & [92] & 24397103 & $\begin{array}{l}\text { Childhood moyamoya disease accompanying Leigh } \\
\text { syndrome }\end{array}$ & Syndrome & Leigh syndrome \\
\hline 75 & [93] & 22759690 & Moyamoya vascular pattern in Alagille syndrome & Syndrome & Alagille syndrome \\
\hline 76 & [94] & 24103673 & $\begin{array}{l}\text { Moyamoya disease associated with asymptomatic } \\
\text { mosaic Turner syndrome: a rare cause of hemorrhagic } \\
\text { stroke }\end{array}$ & Syndrome & Turner syndrome \\
\hline 77 & [95] & 25307898 & Moyamoya syndrome or Behçet's disease? & Syndrome & Behcet's disease \\
\hline 78 & [96] & 25528372 & Moyamoya in a patient with Sneddon's syndrome & Syndrome & Sneddon's syndrome \\
\hline 79 & [97] & 30761079 & $\begin{array}{l}\text { Extracranial and intracranial vasculopathy with } \\
\text { "moyamoya phenomenon" in association with Alagille } \\
\text { syndrome }\end{array}$ & Syndrome & Alagille syndrome \\
\hline 80 & [98] & 28380489 & Moyamoya in a patient with Smith-Magenis syndrome & Syndrome & $\begin{array}{l}\text { Smith-Magenis } \\
\text { syndrome }\end{array}$ \\
\hline 81 & [99] & 26043700 & $\begin{array}{l}\text { Pretransplant management of basilar artery aneurysm } \\
\text { and moyamoya disease in a child with Alagille syndrome }\end{array}$ & Syndrome & Alagille syndrome \\
\hline 82 & [100] & 23692605 & $\begin{array}{l}\text { Moyamoya syndrome in a Malaysian child with Down } \\
\text { syndrome }\end{array}$ & Syndrome & Down syndrome \\
\hline 83 & [101] & 28436815 & Moyamoya in a patient with FIRES: a first case report & Syndrome & FIRES \\
\hline 84 & [102] & 26576049 & $\begin{array}{l}\text { Anesthetic management of a parturient with PHACE } \\
\text { syndrome for cesarean delivery }\end{array}$ & Syndrome & PHACE syndrome \\
\hline 85 & [103] & 26844876 & $\begin{array}{l}\text { Moyamoya disease in a patient with VACTERL } \\
\text { association }\end{array}$ & Syndrome & VACTERL association \\
\hline 86 & [104] & 26540473 & $\begin{array}{l}\text { Morning glory syndrome with carotid and middle cerebral } \\
\text { artery vasculopathy }\end{array}$ & Syndrome & $\begin{array}{l}\text { Morning glory } \\
\text { syndrome }\end{array}$ \\
\hline 87 & [105] & 28859027 & $\begin{array}{l}\text { Subarachnoid hemorrhage revealing moyamoya } \\
\text { syndrome in a patient with May-Hegglin anomaly }\end{array}$ & Syndrome & May-Hegglin anomaly \\
\hline 88 & [106] & 22541517 & ssociation of mesial temporal sclerosis and moyamoya & Syndromes & Mesial temporal \\
\hline
\end{tabular}




\section{Cureus}

\begin{tabular}{|c|c|c|c|c|c|}
\hline \multicolumn{5}{|c|}{ syndrome } & \multirow{2}{*}{$\begin{array}{l}\text { sclerosis } \\
\text { Mesial temporal } \\
\text { sclerosis }\end{array}$} \\
\hline 89 & [107] & 23560742 & Moyamoya disease with mesial temporal sclerosis & Syndrome & \\
\hline 90 & [108] & 24172590 & $\begin{array}{l}\text { Presentation with recurrent intractable headache: a } \\
\text { patient with moyamoya syndrome--case report }\end{array}$ & Headache & Headache \\
\hline 91 & [109] & 31245213 & $\begin{array}{l}\text { A case report of moyamoya disease presenting as } \\
\text { headache in a 35-year-old Hispanic man }\end{array}$ & Headache & Headache \\
\hline 92 & [110] & 29902294 & $\begin{array}{l}\text { Bilateral visual field loss in an adolescent girl with } \\
\text { migraine headaches }\end{array}$ & Headache & Migraine \\
\hline 93 & [111] & 22425396 & $\begin{array}{l}\text { Migraine-like headache and ocular malformations may } \\
\text { herald moyamoya syndrome }\end{array}$ & Headache & Migraine \\
\hline 94 & [112] & 28132315 & O063. Moyamoya disease and headache: case report & Headache & headache \\
\hline 95 & [113] & 23391943 & $\begin{array}{l}\text { Postpneumococcal moyamoya syndrome case report and } \\
\text { review of the postinfective cases }\end{array}$ & Post-infection & Postpneumococcal \\
\hline 96 & [114] & 32434020 & $\begin{array}{l}\text { A rare case of postinfectious moyamoya syndrome: case } \\
\text { report and review of the literature }\end{array}$ & Post-infection & Postinfectious \\
\hline 97 & [115] & 28411258 & $\begin{array}{l}\text { Patient with severe moyamoya disease who presents with } \\
\text { acute cortical blindness }\end{array}$ & Ophthalmologic & $\begin{array}{l}\text { Acute cortical } \\
\text { blindness }\end{array}$ \\
\hline 98 & [116] & 25799075 & $\begin{array}{l}\text { Ocular ischemic syndrome presenting as retinal vasculitis } \\
\text { in a patient with moyamoya syndrome }\end{array}$ & Ophthalmologic & $\begin{array}{l}\text { Ocular ischemic } \\
\text { syndrome }\end{array}$ \\
\hline 99 & [117] & 24515702 & $\begin{array}{l}\text { "The fainted man:" hypoperfusion encephalopathy in a } \\
\text { patient with moyamoya }\end{array}$ & Neurological & $\begin{array}{l}\text { Hypoperfusion } \\
\text { encephalopathy }\end{array}$ \\
\hline 100 & [118] & 30554704 & $\begin{array}{l}\text { Neurologically acquired laryngomalacia in a pediatric } \\
\text { patient with moyamoya: A case report and literature } \\
\text { review }\end{array}$ & Neurological & Laryngomalacia \\
\hline 101 & [119] & 30564539 & $\begin{array}{l}\text { Depression and catatonia: a case of neuropsychiatric } \\
\text { complications of moyamoya disease }\end{array}$ & Neurological & $\begin{array}{l}\text { Neuropsychiatric } \\
\text { complications }\end{array}$ \\
\hline 102 & [120] & 31868692 & $\begin{array}{l}\text { Neuropsychological profile associated with moyamoya } \\
\text { disease: A case report }\end{array}$ & Neurological & $\begin{array}{l}\text { Neuropsychological } \\
\text { profile }\end{array}$ \\
\hline 103 & [121] & 30929500 & $\begin{array}{l}\text { Psychiatric comorbidity in moyamoya disease and } \\
\text { preliminary guidelines for treatment }\end{array}$ & Neurological & $\begin{array}{l}\text { Psychiatric } \\
\text { comorbidity }\end{array}$ \\
\hline 104 & [122] & 29179236 & $\begin{array}{l}\text { Moyamoya syndrome manifesting with choreiform } \\
\text { movements }\end{array}$ & Neurological & $\begin{array}{l}\text { Choreiform } \\
\text { movements }\end{array}$ \\
\hline 105 & $\begin{array}{l}\text { \{Platzen, } \\
2017 \\
\# 1419\}[123]\end{array}$ & 28526173 & $\begin{array}{l}\text { Chorea gravidarum associated with moyamoya } \\
\text { angiopathy treated with alpha-methyldopa }\end{array}$ & Neurological & Chorea gravidarum \\
\hline 106 & $\begin{array}{l}\{\text { Laatar, } \\
2017 \\
\# 1145\}[124]\end{array}$ & 29878297 & $\begin{array}{l}\text { Generalized dystonia : unusual mode of revelation of } \\
\text { moyamoya disease }\end{array}$ & Neurological & Generalized dystonia \\
\hline 107 & [125] & 26450280 & $\begin{array}{l}\text { Pial synangiosis ameliorates movement disorders in the } \\
\text { absence of prior stroke in moyamoya disease }\end{array}$ & Neurological & Movement disorders \\
\hline 108 & [126] & 24022555 & An unusual cause of hemiplegia in a 28-year-old woman & Neurological & Hemiplegia \\
\hline 109 & [127] & 21121731 & $\begin{array}{l}\text { Patients with moyamoya disease presenting with } \\
\text { movement disorder }\end{array}$ & Neurological & Movement disorder \\
\hline 110 & [128] & 27849243 & $\begin{array}{l}\text { Moyamoya disease: impact on the performance of oral } \\
\text { and written language }\end{array}$ & Neurological & $\begin{array}{l}\text { Oral and written } \\
\text { language deficits }\end{array}$ \\
\hline 111 & $\begin{array}{l}\text { ZZalonis, } \\
2010 \\
\# 2694\}[129]\end{array}$ & 21323131 & $\begin{array}{l}\text { Cognitive deficits presenting as psychiatric symptoms in a } \\
\text { patient with moyamoya disease }\end{array}$ & Neurological & Cognitive deficits \\
\hline 112 & [130] & 23793182 & spas & Neurological & Hemifacial spasm \\
\hline
\end{tabular}




\section{Cureus}

\begin{tabular}{|c|c|c|c|c|c|}
\hline & & & disease & & \\
\hline 113 & [131] & 28698731 & $\begin{array}{l}\text { Intermittent hemiplegia in a boy with primary moyamoya } \\
\text { disease: a case report from Iran }\end{array}$ & Neurological & $\begin{array}{l}\text { Intermittent } \\
\text { hemiplegia }\end{array}$ \\
\hline 114 & [132] & 26890714 & $\begin{array}{l}\text { Unilateral sudden hearing loss: a rare symptom of } \\
\text { moyamoya disease }\end{array}$ & Neurological & Hearing loss \\
\hline 115 & [133] & 25982655 & $\begin{array}{l}\text { Transcatheter creation of a reverse Potts shunt in a } \\
\text { patient with severe pulmonary arterial hypertension } \\
\text { associated with moyamoya syndrome }\end{array}$ & Vascular & $\begin{array}{l}\text { Pulmonary arterial } \\
\text { hypertension }\end{array}$ \\
\hline 116 & [134] & 29724978 & $\begin{array}{l}\text { Atypical presentation of moyamoya disease with } \\
\text { pulmonary hypertension: a case report }\end{array}$ & Vascular & $\begin{array}{l}\text { Pulmonary } \\
\text { hypertension }\end{array}$ \\
\hline 117 & [135] & 28946753 & 6-year-old with severe hypertension & Vascular & Hypertension \\
\hline 118 & [136] & 23430230 & $\begin{array}{l}\text { Ophthalmic artery ischemic syndrome associated with } \\
\text { neurofibromatosis and moyamoya syndrome }\end{array}$ & Vascular & $\begin{array}{l}\text { Ophthalmic artery } \\
\text { ischemic syndrome }\end{array}$ \\
\hline 119 & [137] & 20526643 & $\begin{array}{l}\text { Moyamoya disease and arteriovenous fistula of the } \\
\text { epiaortic vessels }\end{array}$ & Vascular & $\begin{array}{l}\text { Arteriovenous fistula } \\
\text { of the epiaortic } \\
\text { vessels }\end{array}$ \\
\hline 120 & [138] & 32705354 & $\begin{array}{l}\text { Central retinal artery occlusion occurring } 30 \text { years after } \\
\text { successful revascularization surgery for moyamoya } \\
\text { disease: case report }\end{array}$ & Vascular & $\begin{array}{l}\text { Central retinal artery } \\
\text { occlusion }\end{array}$ \\
\hline 121 & [139] & 26605691 & Retinal arterial tortuosity in moyamoya disease & Vascular & $\begin{array}{l}\text { Retinal arterial } \\
\text { tortuosity }\end{array}$ \\
\hline 122 & [140] & 27928391 & $\begin{array}{l}\text { A moyamoya patient with bilateral consecutive branch } \\
\text { retinal vein occlusion }\end{array}$ & Vascular & Retinal vein occlusion \\
\hline 123 & [141] & 23394336 & $\begin{array}{l}\text { Intracranial atherosclerotic disease associated with } \\
\text { moyamoya collateral formation: histopathological findings }\end{array}$ & Vascular & $\begin{array}{l}\text { Intracranial } \\
\text { atherosclerotic } \\
\text { disease }\end{array}$ \\
\hline 124 & [142] & 25323944 & $\begin{array}{l}\text { Hemodynamic features of non-aneurysmal subarachnoid } \\
\text { hemorrhage in a case of familial moyamoya disease: a } \\
\text { transcranial Doppler ultrasound study }\end{array}$ & Vascular & $\begin{array}{l}\text { Non-aneurysmal } \\
\text { subarachnoid } \\
\text { hemorrhage }\end{array}$ \\
\hline 125 & [143] & 29367356 & $\begin{array}{l}\text { Multiple anomalies in the origin and course of vertebral } \\
\text { arteries and aberrant right subclavian artery in a child } \\
\text { with moyamoya syndrome }\end{array}$ & Vascular & $\begin{array}{l}\text { Anomalies in the } \\
\text { origin and course of } \\
\text { vertebral arteries and } \\
\text { aberrant right } \\
\text { subclavian artery }\end{array}$ \\
\hline 126 & [144] & 28808729 & $\begin{array}{l}\text { Twig-like middle cerebral artery: a seldom vessel } \\
\text { anomaly of important relevance }\end{array}$ & Vascular & $\begin{array}{l}\text { Twig-like middle } \\
\text { cerebral artery }\end{array}$ \\
\hline 127 & [145] & 20809708 & $\begin{array}{l}\text { Diffuse and uncontrolled vascular smooth muscle cell } \\
\text { proliferation in rapidly progressing pediatric moyamoya } \\
\text { disease }\end{array}$ & Vascular & $\begin{array}{l}\text { Vascular smooth } \\
\text { muscle cell } \\
\text { proliferation }\end{array}$ \\
\hline 128 & [146] & 25773096 & $\begin{array}{l}\text { Central retinal vein occlusion with bilateral stenosis of the } \\
\text { internal carotid arteries }\end{array}$ & Vascular & $\begin{array}{l}\text { Central retinal vein } \\
\text { occlusion }\end{array}$ \\
\hline 129 & [147] & 26864187 & $\begin{array}{l}\text { Peripheral pulmonary artery stenoses in the setting of } \\
\text { moyamoya }\end{array}$ & Vascular & $\begin{array}{l}\text { Peripheral pulmonary } \\
\text { artery stenoses }\end{array}$ \\
\hline 130 & [148] & 32322754 & $\begin{array}{l}\text { Central retinal artery occlusion as initial presentation of } \\
\text { moyamoya disease in a middle-aged woman }\end{array}$ & Vascular & $\begin{array}{l}\text { Central retinal artery } \\
\text { occlusion }\end{array}$ \\
\hline 131 & [149] & 30978680 & $\begin{array}{l}\text { Development of bilateral dural arteriovenous fistulae } \\
\text { following pial synangiosis for moyamoya syndrome: case } \\
\text { report }\end{array}$ & Vascular & $\begin{array}{l}\text { Bilateral dural } \\
\text { arteriovenous fistulae }\end{array}$ \\
\hline 132 & [150] & 30863645 & $\begin{array}{l}\text { ST-elevation myocardial infarction (STEMI) in a patient } \\
\text { with moyamoya disease }\end{array}$ & Vascular & $\begin{array}{l}\text { ST-elevation } \\
\text { myocardial infarction }\end{array}$ \\
\hline 133 & [151] & 22742664 & $\begin{array}{l}\text { Differential diagnosis between cerebral ischemia, focal } \\
\text { seizures and limb shaking TIAs in moyamoya disease }\end{array}$ & Stroke & $\begin{array}{l}\text { Seizures, transient } \\
\text { ischemic attack, } \\
\text { cerebral ischemia }\end{array}$ \\
\hline
\end{tabular}




\section{Cureus}

\begin{tabular}{|c|c|c|c|c|c|}
\hline 134 & [152] & 32733722 & $\begin{array}{l}\text { Postpartum-onset moyamoya disease: a rare cause of } \\
\text { stroke in unexpected }\end{array}$ & Stroke & Postpartum stroke \\
\hline 135 & [153] & 31885602 & $\begin{array}{l}\text { Subacute stroke in a young female: a case of moyamoya } \\
\text { syndrome initially anchoring with anxiety }\end{array}$ & Stroke & Subacute stroke \\
\hline 136 & [154] & 30538867 & $\begin{array}{l}\text { Bilateral cerebrovascular stroke as an initial presenting } \\
\text { symptom of moyamoya disease }\end{array}$ & Stroke & $\begin{array}{l}\text { Bilateral } \\
\text { cerebrovascular } \\
\text { stroke }\end{array}$ \\
\hline 137 & [155] & 32010532 & $\begin{array}{l}\text { A case of delayed diagnosis of moyamoya disease after } \\
\text { recurrent strokes }\end{array}$ & Stroke & Stroke \\
\hline 138 & [156] & 30093460 & $\begin{array}{l}\text { Peek through the smoke: a report of moyamoya disease } \\
\text { in a } 32 \text {-year-old female patient presenting with ischaemic } \\
\text { stroke }\end{array}$ & Stroke & Ischemic stroke \\
\hline 139 & [157] & 22547520 & $\begin{array}{l}\text { Multiple cerebral infarcts in patient with moyamoya } \\
\text { disease }\end{array}$ & Stroke & Stroke \\
\hline 140 & [158] & 27252954 & $\begin{array}{l}\text { Occult moyamoya disease causing fulminant infarction } \\
\text { after septorhinoplasty }\end{array}$ & Stroke & Stroke \\
\hline 141 & [159] & 25496692 & $\begin{array}{l}\text { Endovascular treatment of a ruptured flow aneurysm of } \\
\text { the heubner artery as part of a moyamoya collateral } \\
\text { network in a young patient with an occluded middle } \\
\text { cerebral artery }\end{array}$ & Aneurysm & Fulminant stroke \\
\hline 142 & [160] & 23169511 & $\begin{array}{l}\text { Moyamoya disease misdiagnosed as leptomeningeal } \\
\text { metastases }\end{array}$ & Aneurysm & $\begin{array}{l}\text { Leptomeningeal } \\
\text { metastases }\end{array}$ \\
\hline 143 & [161] & 27236734 & $\begin{array}{l}\text { Ruptured posterior ethmoidal artery aneurysm and } \\
\text { moyamoya disease in an adult patient. Case report }\end{array}$ & Aneurysm & $\begin{array}{l}\text { Ruptured posterior } \\
\text { ethmoidal artery } \\
\text { aneurysm }\end{array}$ \\
\hline 144 & [162] & 20075103 & $\begin{array}{l}\text { Embolization of an unruptured distal lenticulostriate } \\
\text { aneurysm associated with moyamoya disease }\end{array}$ & Aneurysm & $\begin{array}{l}\text { Unruptured distal } \\
\text { lenticulostriate } \\
\text { aneurysm }\end{array}$ \\
\hline 145 & [163] & 29468103 & $\begin{array}{l}\text { Atypical location of intracerebral hemorrhage in } \\
\text { moyamoya disease }\end{array}$ & Hemorrhage & $\begin{array}{l}\text { Intracerebral } \\
\text { hemorrhage }\end{array}$ \\
\hline 146 & [164] & 21748033 & $\begin{array}{l}\text { Non-aneurysmal subarachnoid hemorrhage as } \\
\text { presentation of moyamoya disease in an adult }\end{array}$ & Hemorrhage & $\begin{array}{l}\text { Non-aneurysmal } \\
\text { subarachnoid } \\
\text { hemorrhage }\end{array}$ \\
\hline 147 & [165] & 31689569 & $\begin{array}{l}\text { Pediatric moyamoya presenting as a subarachnoid } \\
\text { hemorrhage from a ruptured anterior cerebral artery } \\
\text { aneurysm }\end{array}$ & Hemorrhage & $\begin{array}{l}\text { Subarachnoid } \\
\text { hemorrhage from a } \\
\text { ruptured anterior } \\
\text { cerebral artery } \\
\text { aneurysm }\end{array}$ \\
\hline 148 & [166] & 20198515 & $\begin{array}{l}\text { Transcranial Doppler ultrasound } \mathrm{CO}_{2} \text { challenge } \\
\text { complicated by subarachnoid hemorrhage in patient with } \\
\text { moyamoya syndrome }\end{array}$ & Hemorrhage & $\begin{array}{l}\text { Subarachnoid } \\
\text { hemorrhage }\end{array}$ \\
\hline 149 & [167] & 31934477 & $\begin{array}{l}\text { Postpartum seizure and subarachnoid haemorrhage } \\
\text { secondary to moyamoya disease }\end{array}$ & Hemorrhage & $\begin{array}{l}\text { Subarachnoid } \\
\text { hemorrhage }\end{array}$ \\
\hline 150 & [168] & 28215908 & $\begin{array}{l}\text { Intraparenchymal haemorrhage secondary to moyamoya } \\
\text { disease in a white patient }\end{array}$ & Hemorrhage & $\begin{array}{l}\text { Intraparenchymal } \\
\text { hemorrhage }\end{array}$ \\
\hline 151 & [169] & 23549955 & $\begin{array}{l}\text { Fatal hemorrhagic stroke in a Caucasian girl with } \\
\text { moyamoya disease }\end{array}$ & Hemorrhage & $\begin{array}{l}\text { Fatal hemorrhagic } \\
\text { stroke }\end{array}$ \\
\hline 152 & [170] & 29482684 & $\begin{array}{l}\text { Dental management of a pediatric patient with moyamoya } \\
\text { syndrome: a rare clinical entity }\end{array}$ & Dental & $\begin{array}{l}\text { Dental caries, stroke } \\
\text { risk }\end{array}$ \\
\hline 153 & [171] & 28814371 & $\begin{array}{l}\text { Dental management considerations in a pediatric patient } \\
\text { with moyamoya disease }\end{array}$ & Dental & $\begin{array}{l}\text { Dental care, stroke } \\
\text { risk }\end{array}$ \\
\hline
\end{tabular}




\section{Cureus}

TABLE 2: List of all papers identified by search criteria, with reference number, PMID, title, and disease/symptom category.

CBL: Casitas B-lineage lymphoma; RNF213: ring finger protein 213; KRIT1: Krev interaction trapped 1; CCM1: cavernous malformations-1; GUCY1A3: guanylate cyclase soluble subunit alpha-3; PHACE: posterior fossa anomalies, hemangioma, arterial anomalies, cardiac anomalies, and eye anomalies; FIRES: febrile infection-related epilepsy syndrome

We categorized the 153 papers into two categories that will be discussed separately. Overall, 44 papers reported a symptom associated with moyamoya, and 109 reported a condition associated with moyamoya.

Symptomatology

Table 3 tabulates symptoms reported in 44 papers describing a moyamoya symptom association.

\begin{tabular}{|l|l|}
\hline Symptom category & $\mathbf{N}(\%)$ \\
\hline Aneurysm & $4(9)$ \\
Dental & $2(5)$ \\
Headache & $5(11)$ \\
Hemorrhage & $7(16)$ \\
Neurologic & $16(36)$ \\
Ophthalmologic & $2(5)$ \\
Stroke & $8(18)$ \\
\hline Total & 44 \\
\hline
\end{tabular}

TABLE 3: List of symptom categories associated with moyamoya.

None of the previously mentioned large database studies reported aneurysms as a primary reported endpoint, possibly because of its rarity in Western patients. One study in Germany published in 2016 of 55 moyamoya patients showed that 37 (67.3\%) had suffered from a headache in the last year, with $47.9 \%$ having migraine-like headaches, of which $58.8 \%$ of the cases described migraine aura $[109,172]$. The rates of hemorrhage reported in several large studies are presented in Table 4.

\begin{tabular}{|c|c|c|c|c|}
\hline Study name & Reference & $\begin{array}{l}\text { Study } \\
\text { duration }\end{array}$ & $\mathbf{N}$ & Findings \\
\hline Characterization of inpatient moyamoya & [17] & 1988-2004 & 2,247 & $\begin{array}{l}11.1 \% \text { hemorrhagic } \\
\text { events }\end{array}$ \\
\hline $\begin{array}{l}\text { Epidemiological and clinical features of patients with moyamoya disease in the } \\
\text { USA }\end{array}$ & [14] & 2005-2008 & 7,473 & $\begin{array}{l}6.1 \% \text { hemorrhagic } \\
\text { strokes }\end{array}$ \\
\hline $\begin{array}{l}\text { Treatment course and outcomes after revascularization surgery for moyamoya } \\
\text { disease in adults }\end{array}$ & [13] & 2013 & 201 & $\begin{array}{l}3.5 \% \text { hemorrhagic } \\
\text { events }\end{array}$ \\
\hline
\end{tabular}

TABLE 4: Rates of hemorrhage in several large studies of moyamoya patients.

In individual moyamoya patients, neurological symptoms secondary to moyamoya included laryngomalacia [118], hypotension encephalopathy [117], behavioral changes [119], oral and written language deficits $[119,128]$, transient psychosis [121,129,173], hemiplegia [126,131], hearing loss [132], and cognitive difficulties [120]. Movement disorders as symptoms of moyamoya included chorea [122,123], dystonia [124,125], hemifacial spasms [130], and hemiballismus [127]. Moreover, Kraemer et al. analyzed the incidence of movement disorders among moyamoya patients in Europe. Using self-rating questionnaires submitted by 59 patients ( 13 males, 36 females), more than half (54.9\%) reported a history of movement 
disorders. Additionally, in response to a multiple-choice question requesting descriptive data on movement disorders, $7 \%$ chose periodic tremors, 33.3\% irregular jerks, $20 \%$ involuntary stiffness and cramps, and $15.7 \%$ unintended movement with loopy or pranced character [174]. Another study examined the clinical records of 143 moyamoya patients ( 89 females, 54 males), of which 16 were Asian, and reported only six cases of choreiform movement disorders (4.2\%) [175]. This wide discrepancy is likely the result of diverging classification schemes.

A case of central deafness in a Caucasian child with moyamoya was reported. The child was a three-year-old male with no known Asian ancestry and was presumed to have suffered developmental retardation because of his cerebrovascular stenosis [176].

Regarding ophthalmologic symptoms, studies have been reported of a moyamoya patient with acute cortical blindness [115] and another with ocular ischemic syndrome [116].

The most commonly reported stroke type was ischemic infarction. In National Inpatient Sample (NIS) studies of disease profiles of moyamoya patients in the United States, ischemic stroke was the most common co-occurring symptom, with an incidence of $20.7 \%$ in the $1988-2004$ study and $11.6 \%$ in the $2005-2008$ study. In the 2013 Nationwide Readmission Database (NRD) study, 5\% of moyamoya patients presenting for revascularization surgery were coded for ischemic stroke.

In the United States, symptomatic moyamoya patients have lower rates of hemorrhagic stroke than their Japanese and Korean counterparts (33.6\% vs. 61\% and 59\%). This supports the notion that moyamoya outside of the West is pathophysiologically distinct from its presentation in East Asia. Additionally, hemorrhagic stroke was more frequent in adults than in children $(18.1 \%$ vs. $1.5 \%, \mathrm{p}<0.05)$ [14]. Further, $20.7 \%$ of patients in the NIS study from 1988 to 2004 were diagnosed with ischemic stroke, $7.4 \%$ with intracerebral hemorrhage, 3.1\% subarachnoid hemorrhage, 3.4\% transient ischemic attack (TIA), $13.5 \%$ anemia, and $13.6 \%$ with sickle cell disease or trait [17]. A higher percentage of moyamoya patients in the West have an identifiable underlying condition than their East Asian counterparts [177].

Additionally, the 2018 study of moyamoya patients who underwent revascularization surgery found that vascular risk factors were common, with one out of four having diabetes, half having hypertension, and $40 \%$ each having hypocholesteremia or a history of smoking. Further, moyamoya has also been reported in patients with fibromuscular dysplasia [178]. Renovascular hypertension has also been reported in association with moyamoya [179].

Disease Associations

Table 5 lists the disease categories associated with moyamoya. 


\section{Cureus}

\begin{tabular}{|l|l|}
\hline Disease category & $\mathbf{N}(\%)$ \\
\hline Autoimmune & $10(9)$ \\
Congenital & $13(12)$ \\
Drugs & $3(3)$ \\
Hematologic & $16(15)$ \\
Metabolic & $5(5)$ \\
Neurocutaneous & $12(11)$ \\
Physical trauma & $3(3)$ \\
Postinfectious & $2(2)$ \\
Radiation therapy & $6(6)$ \\
Renal & $2(2)$ \\
\hline Syndromes & $19(17)$ \\
Vascular & $18(17)$ \\
\hline Total & 109 \\
\hline
\end{tabular}

TABLE 5: Disease categories associated with moyamoya.

We found 10 (9\%) papers describing an association between moyamoya and an autoimmune condition. This finding is consistent with previous reports of associations between MMS and autoimmune diseases in Eastern cohorts [180,181]. In 2012, one research group at the Mayo Clinic in Rochester reported an unusually high prevalence of autoimmune diseases among their cohort of mostly white, mid-Western patients. Later, in 2016, another group in Washington reported four cases of MMS with pre-existing type 1 diabetes mellitus. Two of the patients also had Graves' disease, and another had systemic lupus erythematosus [52]. Then, in 2017, researchers analyzed 2,633 patients in the NIS dataset from 2009 to 2012 and reported a strong ( $<$ < 0.05 ) association between adult-onset autoimmune diseases and moyamoya (NIS lacks distinction between MMS and MMD) in the pediatric population (but not the adult population). The adult-onset autoimmune diseases included Addison's disease, dermatomyositis, granulomatosis with polyangiitis, Graves' disease, multiple sclerosis, myasthenia gravis, polymyositis, primary systemic vasculitis, rheumatoid arthritis, Sjogren's disease, systemic lupus erythematosus, systemic sclerosis, and thyroiditis. By contrast, juvenileonset autoimmune diseases, including diabetes mellitus type 1 and juvenile rheumatoid arthritis, were associated with moyamoya in both the pediatric and adult populations [15]. Finally, in 2018, 26\% of 31 mostly Caucasian MMD patients in Kentucky were reported to have a co-existing autoimmune condition, including rheumatoid arthritis, lupus, hypothyroidism, psoriasis, polyglandular autoimmune type 1, autoimmune hepatitis, Addison's disease, immune thrombocytopenic purpura, Crohn's disease, multiple sclerosis, celiac sprue, and dermatitis herpetiformis [182]. Other reports have shown an association between moyamoya and febrile infection-related epilepsy syndrome (FIRES) [101], Graves' disease [182], and collagenous colitis [183]. Of note, not a single case of co-existing moyamoya and Sjogren's syndrome could be found for a Western patient.

The association between autoimmunity and moyamoya has been thoroughly demonstrated using animal models. Half a century ago, researchers noticed that moyamoya patients were experiencing leptospirosis complications, in which $81.4 \%$ of their cerebral spine fluid had a positive immune response. Later, in 1983 , Japanese researchers observed that moyamoya patients were at a greater risk for tonsillitis, otitis media, maxillary sinusitis, and fever and infection of unknown origin. However, more recent research has called into question the proposed autoimmune etiology of the moyamoya phenotype [184].

We found several reports showing an association between moyamoya and Majewski osteodysplastic primordial dwarfism type 2 (MOPD2) [69-71,185], a rare autosomal recessive disorder. Two studies demonstrated this as well. One study found 11 cases of moyamoya in the literature out of 58 mostly Western patients (19\%) with MOPD2 in 2004 [183], while another found 13 (52\%) among 25 patients with MOPD2 [186]. Moyamoya has been reported in Western patients in association with other rare growth disorders, including Seckel syndrome [187,188] Cockayne syndrome [189], floating harbor syndrome [190], and Noonan syndrome [91]. 
United States [192]. Additionally, it has been reported in association with Alagille syndrome [93,193], a rare, autosomal dominant hepatic disorder. A few cases of moyamoya have been reported in patients with Turner's syndrome $[94,193]$. In our literature search, we found one case of moyamoya with Aicardi Goutieres syndrome [194].

We found three (3\%) papers describing an association between moyamoya and drugs. Oral contraceptives have been recognized as a potential risk factor for moyamoya in the West since at least 1984 [195]. A small cohort study conducted in Canada and the United States consisting of 39 patients with moyamoya [196] and another literature review of moyamoya cases in the United States [183], both in 1997, showed that oral contraceptive use was associated with moyamoya. We found one report of cocaine abuse associated with moyamoya [21]. Another two articles reported an association with nilotinib, a second-generation tyrosine kinase inhibitor [23], and bevacizumab [22].

We found 16 (15\%) papers describing an association between moyamoya and hematologic disorders. These include hereditary spherocytosis [124-127], beta-thalassemia [128,133,173], Fanconi anemia [78], sickle cell anemia [79] and trait [85,86], hemophilia A [131,197], hemoglobin Southamptom [132], and HenochSchonlein purpura [174].

Sickle cell anemia predisposes patients to internal carotid artery stenosis, leading to moyamoya [198]. A 2019 study identified 61 studies in the literature on sickle cell-associated moyamoya revascularization surgery outcomes, presumably a significant number of which occurred in the Western population [199]. A 2011 NIS study of moyamoya admissions from 1988 to 2004 showed that $13.6 \%$ of all moyamoya patients had sickle cell disease or trait at admission [17].

To our knowledge, five cases of MMS with hereditary spherocytosis have been reported [75], of which at least three were Western patients.

Additionally, East Asian researchers have reported at least five cases of moyamoya associated with paroxysmal nocturnal hemoglobinuria [200], although to our knowledge, none have been reported to date in Western patients. Additional hematological conditions not identified by our search criteria included hemoglobin Fairfax-beta-thalassemia in 2008 in Indiana [201] and hemoglobin E/beta-thalassemia in 2009 in Cambodia [202].

We found five papers (5\%) that described an association between moyamoya and metabolic disorders, including mucolipidosis II [36], protein S deficiency [37], CD59 deficiency [38], severe hyperhomocysteinemia [39], and lysinuric protein intolerance [40].

We found 12 (11\%) papers describing an association between moyamoya and Neurocutaneous disorders, including one case of tuberous sclerosis [35] and the rest of neurofibromatosis type 1 (NF1) [24-34]. One study found approximately 250 children with NF1 in the literature since 1976 [12], and another NIS study found 51 of 2,247 moyamoya patients with NF between 1988 and 2004 [17]. NF1 is an autosomal dominant genetic neurocutaneous disorder caused by a mutation in the NF1 gene, located on chromosome 17 (17q11.2). The disorder leads to a large spectrum of central nervous system manifestations, including learning disabilities, mental retardation, seizures, attention deficit with hyperkinesia disorder, neurofibromas, and optic nerve glioma. While the mechanism by which NF1 leads to moyamoya is unknown, it is probably related to the function of the protein encoded by the NF1 gene, a negative regulator of Ras. This is especially plausible because moyamoya is known to be associated with RASopathies, including Noonan syndrome and Costello syndrome.

Associations between tumors and moyamoya have been reported in East Asian literature, but they are very rare [203]. Furthermore, we found two (2\%) papers that described an association between moyamoya and a post-infection state, including meningitis secondary to Aspergillus fumigatus and Escherichia coli [114] and pneumococcal meningitis [113].

On further analysis, we found several other reports of post-infectious moyamoya, including quaternary neurosyphilis in 1989 [204] and Haemophilus influenaze type C meningitis in Texas in 2003 [205]. Moreover, one national multicenter study identified five South African children (four girls of indigenous African ancestry) with human immunodeficiency virus-associated vasculopathy and MMS [206], and a Canadian and US cohort demonstrated an association between moyamoya and tuberculosis in 1997 [196]. Finally, a patient with acquired immunodeficiency syndrome was reported as presenting concurrently with moyamoya [207].

We found six (6\%) papers describing an association between moyamoya and radiation therapy. Radiation therapy is a known risk factor for the development of cerebrovascular pathologies. A recent 2019 PubMed analysis of case reports of radiation-induced moyamoya found 54 reported cases [208]. Proton beam therapy, a safer alternative to traditional radiation therapy, has also been associated with moyamoya [55]. One of the first reported cases of moyamoya associated with radiation therapy in the United States was published in 1978 [209], followed by what was probably the first cohort study of the association in Western patients in Toronto, Ontario in 1993 with five patients treated for optic gliomas [210]. All patients were 
Caucasian, and four received 5,000 rad of radiation or more and one received 2,500 rad. In four cases, the presentation was ischemic and two patients presented with TIAs. While there have been several recent meta-analyses of the literature regarding the association between radiation therapy and moyamoya in the global population, there have been none published to date specifically for Western populations to our knowledge.

We found 19 (17\%) papers that described an association between moyamoya and rare syndromes, including oculoectodermal syndrome [89], Down syndrome [90, 100], Noonan syndrome [91], Leigh syndrome [92], Alagille syndrome [93,97,99], Turner syndrome [94], Behcet's disease [95], Sneddon's syndrome [96], SmithMagenis syndrome [98], FIRES [101], PHACE syndrome [102], VACTERL association [103], Morning Glory syndrome [104], May-Hegglin anomaly [105], and mesial temporal sclerosis syndrome [106,107].

Specifically, the association between Down syndrome and moyamoya has been known for some time [211,212], with as many as 80 distinct cases, excluding large population analyses, being reported in the literature [213]. Although the pathophysiology of this association is not well understood, it might be related to developmental vascular anomalies [212], especially regarding proteins involved in vascular physiology that are encoded in chromosome 21 , such as cystathionine B-synthetase, interferon-gamma receptor, superoxide dismutase, and chains of collagen type VI. Antiphospholipid antibodies have also been found in both moyamoya and Down syndrome and may reflect a possible link between these two pathologic entities [90]. Moreover, patients with Down syndrome have a higher prevalence of autoimmune disorders and autoantibodies, similar to moyamoya, which has been noted for its association with autoimmune disorders, and prevalence of antiphospholipid antibodies [16].

However, less is known about the particular Western expression and prevalence of moyamoya in association with Down syndrome $[90,213,214]$. In probably the first large analysis in Western cohorts, Kainth et al. calculated the prevalence of 3,760 Down syndrome diagnoses in every 100,000 moyamoya cases (approximately 3.8\%) using the NIS dataset of admissions between 2002 and 2009 [16]. Given that the estimated prevalence of Down syndrome among live births from 2004 to 2006 previously calculated by Parker et al. was 14.47 per 100,000 [215], moyamoya patients are estimated to have a 26-fold increased prevalence of Down syndrome relative to the general population. Moreover, the prevalence of Down syndrome among patients admitted with moyamoya who were $<15$ years of age was found to be $9.5 \%$ or 9,540 per 100,000 live births. Finally, the incidence of moyamoya among Down syndrome patients was approximately three times higher than the general population [214].

There appears to be a nuanced difference between the demographic presentation of moyamoya in Down syndrome and the typical presentation. First, the percentage prevalence of Down syndrome among moyamoya patients appears to be the highest in white (65.4\% vs. $47.4 \%)$ and Hispanic patients (14.6\% vs. $10.4 \%)$ and especially low among blacks (10.7\% vs. $25.9 \%)$. The percentage prevalence among females with Down syndrome-associated moyamoya is lower than non-Down syndrome-associated moyamoya ( $58.4 \%$ vs. $70.5 \%)$. Moreover, patients with moyamoya who have Down syndrome are at a greater risk for ischemic symptoms and at a lesser risk for hemorrhagic strokes $(\mathrm{p}<0.05)$. On average, they experience a hospital admission at a younger age (16.2 vs. 33) [16], despite the fact that their moyamoya initially presents at an older age (8.4 vs. 6.5) [213]. These patients more often achieve better neurologic outcomes after surgical revascularization than non-Down syndrome-associated moyamoya (as reflected in improved modified Rankin scale scores in $97 \%$ of cases and lack of post-discharge strokes), despite more often being symptomatic (100\% vs. $75 \%$ ) and experiencing surgical complications (perioperative stroke rate per hemisphere $5.9 \%$ vs. $4 \%$, and perioperative seizure high at $16 \%$ ). Down syndrome-associated moyamoya patients are reported to have strokes more often than non-Down syndrome moyamoya patients (87\% vs 68\%) and more often experience seizures before diagnosis (26\% vs. $6 \%$ ) [90].

Demographics

The results of three studies using the NIS, the largest publicly available, all-payer inpatient database in the United States that represents $20 \%$ of all annual US hospital admissions, are presented in Table 5 . 


\section{Cureus}

\begin{tabular}{|c|c|c|c|}
\hline Race/Admission Years & 1988-2004 [17] & 2002-2008 [12] & 2005-2008 [14] \\
\hline White & 35.4 & 49 & 48.7 \\
\hline African American & 19.7 & 24 & 24.72 \\
\hline Asian/Pacific Islanders & 8.3 & 11 & N/A \\
\hline Hispanic & 5.6 & 11 & 10.5 \\
\hline Native American & 1.4 & N/A & N/A \\
\hline Other & N/A & 5 & 15.9 \\
\hline
\end{tabular}

TABLE 6: Demographic Data (as \%) of US Moyamoya Patients from Three NIS Studies.

Both Japanese and US literature have reported bimodal age distribution of moyamoya prevalence, with peaks in the first and third-to-fourth decades [202]. Additionally, from 1988 to 2004, African Americans accounted for the majority of patients under 19 years of age, which might be an artifact of sickle cell disease, which has a known association with moyamoya. Finally, in a study of moyamoya patients from 1987 to 1998, racial and ethnic disparities between hemispheres appeared to have been maintained in immigrant populations in the United States [186], supporting a genetic pathophysiologic etiology as the classic cause of moyamoya.

In a more recent NIS study using admission dates from 2008 to 2015, incidence of moyamoya in the US population was shown to be increasing and greatest for low-income, urban-living, female (estimated difference of $0.237, \mathrm{p}<0.05$ ), and 18 -44-year-old patients. Incidence rates for ethnicities were 0.509 for Asian/Pacific Islanders, 0.292 for blacks, 0.148 for whites, and 0.121 for Hispanics.

Estimates for relative rates of moyamoya between biological sexes range from $61.9 \%$ to $75 \%$ among females. However, an NIS study found that moyamoya patients younger than 18 from 1988 to 2004 displayed a male predominance before 1998 and that the rates of moyamoya among females significantly increased after 1994 for ages 36-55. Interestingly, the study also found that among African Americans, there was no significant female predominance [17].

\section{Discussion}

This paper presents the first attempt at a scoping review of the Western presentation of MMS. Moyamoya is a unique angiographic finding, characterized by stenosis of the supraclinoid internal carotid artery, middle carotid artery, anterior carotid artery, or their branches, followed by extensive collateralization of the cerebral vasculature at the base of the skull. It has historically been considered endemic to Asian populations, especially Japananese and Korean, where most cases have been found. However, a significant minority of patients in the West have presented with moyamoya findings lacking an obvious Asian heritage. Motivations for compiling this report include: (1) prevalence rate of moyamoya has been rising in recent years [14] (although possibly due to improved diagnostics and awareness [13] rather than increased prevalence of pathophysiologic mechanisms); (2) the western phenotype has been known for some time to diverge from its Asian counterpart; and (3) moyamoya has been associated with significant risk for cerebrovascular accidents. Elucidating the disease profile can provide insights into the unique pathophysiology of moyamoya and provide the groundwork for therapeutics.

We searched PubMed for case reports on moyamoya published in the last 10 years, which resulted in 725 papers. From these, we selected 357 English-language papers that reported a moyamoya association (with moyamoya as either etiology or symptom). We then selected 153 papers by excluding those reporting on patients from Asian populations. This selection was not straightforward as patients' heritage and location of presentation were often not available. At the risk of misidentifying a few reports and in the interest of consistency, simplicity, and efficiency, we chose to categorize the patients' East-West localization based on the location of the lead institution from which the paper was published.

Symptoms of note were, in order of prevalence in reports, neurological, stroke, hemorrhage, headache, aneurysm, dental, and ophthalmologic. Further review of the literature revealed that neurological symptoms associated with moyamoya include laryngomalacia, hypotension encephalopathy, behavioral changes, language deficits, transient psychosis, hemiplegia and movement disorders, hearing loss, and cognitive difficulties. Vascular symptoms (excluding specific markers of moyamoya, for example, occlusion of anterior, middle cerebral, and internal carotid arteries) included vertebral artery, carotid artery, ophthalmic artery, retinal artery and vein, pulmonary artery occlusions, retinal artery tortuosity, arteriovenous fistula, and vascular smooth muscle proliferation. Ophthalmologic symptoms included acute cortical blindess, ocular ischemic syndrome, and ocular malformations. 
In several large analyses of multi-year NIS data, ischemic stroke was the most common symptom occurring in association with moyamoya, followed by hemorrhage, subarachnoid hemorrhage, TIA, anemia, and sickle cell disease.

Roughly two-thirds of western moyamoya patients were females, with numbers from NIS studies of moyamoya patients ranging from $62 \%$ to $72 \%$, and a slightly higher ratio for moyamoya patients presenting for revascularization surgery (74\%). The female-to-male ratio appears to be rising, with $61.9 \%$ female cases reported during 1988-2004 and 70.5\% during 2002-2009.

Common disease profiles of moyamoya patients include, in order of prevalence in reports, syndromes, vascular, congenital, hematologic, neurocutaneous, autoimmune, radiation therapy, metabolic, physical trauma, drugs, and post-infectious pathologies. Vascular comorbidities were especially common, with one 2018 study of revascularization moyamoya patients reporting that one of four presented with diabetes, half with hypertension, and $40 \%$ for both hypercholesterolemia and smoking.

Of note, Down syndrome, a common condition associated with moyamoya, is more prevalent in Caucasian and Hispanic populations, and less common in women relative to the non-Down syndrome moyamoya population. Down syndrome patients with moyamoya are diagnosed with moyamoya at an older age but present at a hospital at a younger age and are more symptomatic (especially strokes and seizures), although they have better neurologic outcomes after revascularization surgery than non-Down syndrome moyamoya patients. Additionally, tumor-associated moyamoa appears to be a rare occurrence.

Autoimmune conditions commonly occur in association with moyamoya, and we found nine reports in our literature serac (10\%). This is not surprising given that moyamoya has long been thought to have a pathophysiologic relationship with autoimmunity. This has been supported by half a century of research, although more recent findings have called this paradigm into question [184].

Limitations to our review include the inconsistency of reports included in our analysis, such that some reports may have been inappropriately excluded and that those included might not be representative of the overall prevalence of Western patients. Additionally, using the location of the first author as a surrogate for the patient's heritage has obvious limitations as an immigrant or descendent of immigrants would not freely share ethnicity. Moreover, generalized disease and symptom profiles serve as only a guide for future research but cannot provide specific extrapolations for individual presentations.

\section{Conclusions}

Our scoping review of the relevant Western literature on moyamoya has revealed an interesting panoply of demographic data, symptomatology, and associated diseases. Moyamoya is part of a complex web of interconnected disease processes that culminate in a unique radiographic finding. Moreover, while moyamoya is often thought of as native to East Asia, it presents often enough in the West that it can be understood as a unique moyamoya phenotype. Here, we aimed to collate literature reports on moyamoya presentation in the West to better appreciate and understand the unique Western moyamoya phenotype. We hope that this information guides treatment planning as moyamoya findings can herald the advent or concurrence of potentially fatal disease processes. Additionally, highlighting the unique characteristics of the Western moyamoya presentation can help further guide researchers to important discoveries in moyamoya pathophysiologic pathways and sequelae. This could, in turn, translate into discoveries of more effective treatment targets and therapeutic developments. Further research is warranted in the form of rigorously conducted systematic reviews to better contrast the Eastern and Western moyamoya phenotypes to stratify treatment planning with a greater degree of granularity.

\section{Appendices}




\section{Cureus}

Preferred Reporting Items for Systematic reviews and Meta-Analyses extension for Scoping Reviews (PRISMA-ScR) Checklist

\begin{tabular}{|c|c|c|c|}
\hline SECTION & ITEM & PRISMA-ScR CHECKLIST ITEM & $\begin{array}{l}\text { REPORTED } \\
\text { ON PAGE \# }\end{array}$ \\
\hline \multicolumn{4}{|l|}{ TITLE } \\
\hline Title & 1 & Identify the report as a scoping review. & Title \\
\hline \multicolumn{4}{|l|}{ ABSTRACT } \\
\hline $\begin{array}{l}\text { Structured } \\
\text { summary }\end{array}$ & 2 & $\begin{array}{l}\text { Provide a structured summary that includes (as } \\
\text { applicable): background, objectives, eligibility criteria, } \\
\text { sources of evidence, charting methods, results, and } \\
\text { conclusions that relate to the review questions and } \\
\text { objectives. }\end{array}$ & Abstract \\
\hline \multicolumn{4}{|l|}{ INTRODUCTION } \\
\hline Rationale & 3 & $\begin{array}{l}\text { Describe the rationale for the review in the context of } \\
\text { what is already known. Explain why the review } \\
\text { questions/objectives lend themselves to a scoping } \\
\text { review approach. }\end{array}$ & $\begin{array}{l}\text { Introduction } \\
\text { and } \\
\text { Background }\end{array}$ \\
\hline Objectives & 4 & $\begin{array}{l}\text { Provide an explicit statement of the questions and } \\
\text { objectives being addressed with reference to their key } \\
\text { elements (e.g., population or participants, concepts, } \\
\text { and context) or other relevant key elements used to } \\
\text { conceptualize the review questions and/or objectives. }\end{array}$ & $\begin{array}{l}\text { Introduction } \\
\text { and } \\
\text { Background }\end{array}$ \\
\hline \multicolumn{4}{|c|}{ 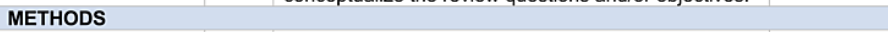 } \\
\hline $\begin{array}{l}\text { Protocol and } \\
\text { registration }\end{array}$ & 5 & $\begin{array}{l}\text { Indicate whether a review protocol exists; state if and } \\
\text { where it can be accessed (e.g., a Web address); and } \\
\text { if available, provide registration information, including } \\
\text { the registration number. }\end{array}$ & Methods \\
\hline Eligibility criteria & 6 & $\begin{array}{l}\text { Specify characteristics of the sources of evidence } \\
\text { used as eligibility criteria (e.g., years considered, } \\
\text { language, and publication status), and provide a } \\
\text { rationale. }\end{array}$ & Methods \\
\hline $\begin{array}{l}\text { Information } \\
\text { sources* }^{*}\end{array}$ & 7 & $\begin{array}{l}\text { Describe all information sources in the search (e.g., } \\
\text { databases with dates of coverage and contact with } \\
\text { authors to identify additional sources), as well as the } \\
\text { date the most recent search was executed. }\end{array}$ & Methods \\
\hline Search & 8 & $\begin{array}{l}\text { Present the full electronic search strategy for at least } \\
1 \text { database, including any limits used, such that it } \\
\text { could be repeated. }\end{array}$ & Methods \\
\hline $\begin{array}{l}\text { Selection of } \\
\text { sources of } \\
\text { evidencet }\end{array}$ & 9 & $\begin{array}{l}\text { State the process for selecting sources of evidence } \\
\text { (i.e., screening and eligibility) included in the scoping } \\
\text { review. }\end{array}$ & Methods \\
\hline $\begin{array}{l}\text { Data charting } \\
\text { process } \neq\end{array}$ & 10 & $\begin{array}{l}\text { Describe the methods of charting data from the } \\
\text { included sources of evidence (e.g., calibrated forms or } \\
\text { forms that have been tested by the team before their } \\
\text { use, and whether data charting was done } \\
\text { independently or in duplicate) and any processes for } \\
\text { obtaining and confirming data from investigators. }\end{array}$ & Methods \\
\hline Data items & 11 & $\begin{array}{l}\text { List and define all variables for which data were } \\
\text { sought and any assumptions and simplifications } \\
\text { made. }\end{array}$ & Methods \\
\hline $\begin{array}{l}\text { Critical appraisal of } \\
\text { individual sources } \\
\text { of evidence§ }\end{array}$ & 12 & $\begin{array}{l}\text { If done, provide a rationale for conducting a critical } \\
\text { appraisal of included sources of evidence; describe } \\
\text { the methods used and how this information was used } \\
\text { in any data synthesis (if appropriate). }\end{array}$ & N/A \\
\hline
\end{tabular}




\section{Cureus}

\begin{tabular}{|c|c|c|c|}
\hline SECTION & ITEM & PRISMA-ScR CHECKLIST ITEM & $\begin{array}{l}\text { REPORTED } \\
\text { ON PAGE \# }\end{array}$ \\
\hline $\begin{array}{l}\text { Synthesis of } \\
\text { results }\end{array}$ & 13 & $\begin{array}{l}\text { Describe the methods of handling and summarizing } \\
\text { the data that were charted. }\end{array}$ & Methods \\
\hline \multicolumn{4}{|l|}{ RESULTS } \\
\hline $\begin{array}{l}\text { Selection of } \\
\text { sources of } \\
\text { evidence }\end{array}$ & 14 & $\begin{array}{l}\text { Give numbers of sources of evidence screened, } \\
\text { assessed for eligibility, and included in the review, } \\
\text { with reasons for exclusions at each stage, ideally } \\
\text { using a flow diagram. }\end{array}$ & Results \\
\hline $\begin{array}{l}\text { Characteristics of } \\
\text { sources of } \\
\text { evidence }\end{array}$ & 15 & $\begin{array}{l}\text { For each source of evidence, present characteristics } \\
\text { for which data were charted and provide the citations. }\end{array}$ & Results \\
\hline $\begin{array}{l}\text { Critical appraisal } \\
\text { within sources of } \\
\text { evidence }\end{array}$ & 16 & $\begin{array}{l}\text { If done, present data on critical appraisal of included } \\
\text { sources of evidence (see item 12). }\end{array}$ & $N / A$ \\
\hline $\begin{array}{l}\text { Results of } \\
\text { individual sources } \\
\text { of evidence }\end{array}$ & 17 & $\begin{array}{l}\text { For each included source of evidence, present the } \\
\text { relevant data that were charted that relate to the } \\
\text { review questions and objectives. }\end{array}$ & Results \\
\hline $\begin{array}{l}\text { Synthesis of } \\
\text { results }\end{array}$ & 18 & $\begin{array}{l}\text { Summarize and/or present the charting results as they } \\
\text { relate to the review questions and objectives. }\end{array}$ & Results \\
\hline \multicolumn{4}{|l|}{ DISCUSSION } \\
\hline $\begin{array}{l}\text { Summary of } \\
\text { evidence }\end{array}$ & 19 & $\begin{array}{l}\text { Summarize the main results (including an overview of } \\
\text { concepts, themes, and types of evidence available), } \\
\text { link to the review questions and objectives, and } \\
\text { consider the relevance to key groups. }\end{array}$ & Discussion \\
\hline Limitations & 20 & Discuss the limitations of the scoping review process. & Discussion \\
\hline Conclusions & 21 & $\begin{array}{l}\text { Provide a general interpretation of the results with } \\
\text { respect to the review questions and objectives, as well } \\
\text { as potential implications and/or next steps. }\end{array}$ & Conclusion \\
\hline \multicolumn{4}{|c|}{. } \\
\hline Funding & 22 & $\begin{array}{l}\text { Describe sources of funding for the included sources } \\
\text { of evidence, as well as sources of funding for the } \\
\text { scoping review. Describe the role of the funders of the } \\
\text { scoping review. }\end{array}$ & Funding \\
\hline \multicolumn{4}{|c|}{ 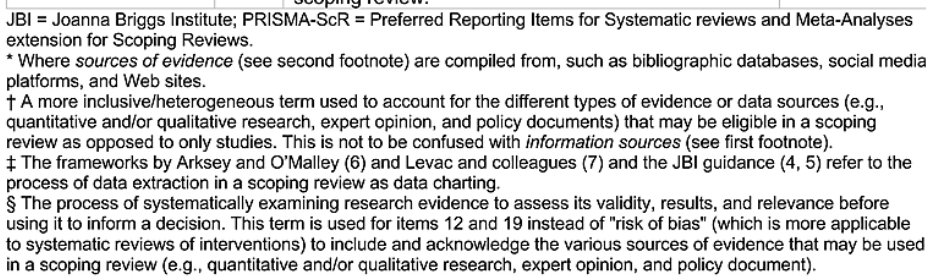 } \\
\hline
\end{tabular}

FIGURE 3: Preferred Reporting Items for Systematic Reviews and MetaAnalyses extension for scoping reviews (PRISMA-ScR) page 2.

\section{Additional Information \\ Disclosures}

Conflicts of interest: In compliance with the ICMJE uniform disclosure form, all authors declare the following: Payment/services info: All authors have declared that no financial support was received from any organization for the submitted work. Financial relationships: David J. Altschul declare(s) Consultation work from Stryker. David J. Altschul declare(s) Consultation work from Microinvention. Other relationships: All authors have declared that there are no other relationships or activities that could appear to have influenced the submitted work.

\section{References}

1. Suzuki J, Takaku A: Cerebrovascular "moyamoya" disease. Disease showing abnormal net-like vessels in base of brain. Arch Neurol. 1969, 20:288-99. 10.1001/archneur.1969.00480090076012

2. Kim JM, Lee SH, Roh JK: Changing ischaemic lesion patterns in adult moyamoya disease . J Neurol Neurosurg Psychiatry. 2009, 80:36-40. 10.1136/jnnp.2008.145078

3. Suzuki J, Kodama N: Cerebrovascular "moyamoya" disease: second report: collateral routes to forebrain via ethmoid sinus and superior nasal meatus. Angiology. 1971, 22:223-36. 10.1177/000331977102200408

4. Kamada F, Aoki Y, Narisawa A, et al.: A genome-wide association study identifies RNF213 as the first moyamoya disease gene. J Hum Genet. 2011, 56:34-40. 10.1038/jhg.2010.132 
5. Ito A, Fujimura M, Niizuma K, et al.: Enhanced post-ischemic angiogenesis in mice lacking RNF213; a susceptibility gene for moyamoya disease. Brain Res. 2015, 1594:310-20. 10.1016/j.brainres.2014.11.014

6. Ohkubo K, Sakai Y, Inoue H, et al.: Moyamoya disease susceptibility gene RNF213 links inflammatory and angiogenic signals in endothelial cells. Sci Rep. 2015, 5:13191. 10.1038/srep13191

7. Liu W, Morito D, Takashima S, et al.: Identification of RNF213 as a susceptibility gene for moyamoya disease and its possible role in vascular development. PLoS One. 2011, 6:e22542. 10.1371/journal.pone.0022542

8. Zhang Q, Liu Y, Yu L, et al.: The association of the RNF213 p.R4810K polymorphism with quasi-moyamoya disease and a review of the pertinent literature. World Neurosurg. 2017, 99:701-8. 10.1016/j.wneu.2016.12.119

9. Milewicz DM, Østergaard JR, Ala-Kokko LM, et al.: De novo ACTA2 mutation causes a novel syndrome of multisystemic smooth muscle dysfunction. Am J Med Genet A. 2010, 152A:2437-43. 10.1002/ajmg.a.33657

10. Scott RM, Smith ER: Moyamoya disease and moyamoya syndrome. N Engl J Med. 2009, 360:1226-37. 10.1056/NEJMra0804622

11. Chen JB, Liu Y, Zhou LX, Sun H, He M, You C: Prevalence of autoimmune disease in moyamoya disease patients in Western Chinese population. J Neurol Sci. 2015, 351:184-6. 10.1016/j.jns.2015.02.037

12. Starke RM, Crowley RW, Maltenfort M, et al.: Moyamoya disorder in the United States. Neurosurgery. 2012, 71:93-9. 10.1227/NEU.0b013e318253ab8e

13. Kahn A, Kaur G, Stein L, Tuhrim S, Dhamoon MS: Treatment course and outcomes after revascularization surgery for moyamoya disease in adults. J Neurol. 2018, 265:2666-71. 10.1007/s00415-018-9044-z

14. Kainth D, Chaudhry SA, Kainth H, Suri FK, Qureshi AI: Epidemiological and clinical features of moyamoya disease in the USA. Neuroepidemiology. 2013, 40:282-7. 10.1159/000345957

15. Mejia-Munne JC, Ellis JA, Feldstein NA, Meyers PM, Connolly ES: Moyamoya and inflammation. World Neurosurg. 2017, 100:575-8. 10.1016/j.wneu.2017.01.012

16. Kainth DS, Chaudhry SA, Kainth HS, Suri FK, Qureshi AI: Prevalence and characteristics of concurrent Down syndrome in patients with moyamoya disease. Neurosurgery. 2013, 72:210-5. 10.1227/NEU.0b013e31827b9beb

17. Lee DJ, Liebeskind DS: Characterization of inpatient moyamoya in the United States: 1988-2004. Front Neurol. 2011, 2:43. 10.3389/fneur.2011.00043

18. Ghaffari-Rafi A, Ghaffari-Rafi S, Leon-Rojas J: Socioeconomic and demographic disparities of moyamoya disease in the United States. Clin Neurol Neurosurg. 2020, 192:105719. 10.1016/j.clineuro.2020.105719

19. Hong JC, Ramos E, Copeland CC, Ziv K: Transient intraoperative central diabetes insipidus in moyamoya patients undergoing revascularization surgery: a mere coincidence?. A A Case Rep. 2016, 6:224-7. 10.1213/xaa.0000000000000287

20. Romão EA, Lourenço CM, Júnior WM, et al.: What lies beneath: Fabry nephropathy in a female patient with severe cerebrovascular disease. Clin Nephrol. 2013, 79:330-4. 10.5414/cn107193

21. Unger MD, Georges J, Shaikh HA, Kavi T: Moyamoya tipping point: fatal bilateral MCA territory infarction following cocaine abuse. BMJ Case Rep. 2018, 2018:bcr-2017-222883. 10.1136/bcr-2017-222883

22. Ullrich NJ, Zimmerman M, Smith E, Irons M, Marcus K, Kieran MW: Association of rapidly progressive moyamoya syndrome with bevacizumab treatment for glioblastoma in a child with neurofibromatosis type 1. J Child Neurol. 2011, 26:228-30. 10.1177/0883073810379639

23. Zhuo DX, Ragosta M III, Patterson B: Tyrosine kinase inhibitor toxicity manifesting as comorbid moyamoya syndrome and obstructive coronary artery disease: a case report and review of the literature. Catheter Cardiovasc Interv. 2019, 94:117-9. 10.1002/ccd.28189

24. Budişteanu M, Burloiu CM, Papuc SM, Focşa IO, Riga D, Riga S, Arghir A: Neurofibromatosis type 1 associated with moyamoya syndrome. Case report and review of the literature. Rom J Morphol Embryol. 2019, 60:713-6.

25. Delvoye F, Hervé D, Chabriat H, Mawet J: Moyamoya syndrome related to neurofibromatosis of type 1: a case report. Acta Neurol Belg. 2013, 113:539-41. 10.1007/s13760-013-0250-2

26. Novara S, Singh S, Rashid S: "Ivy sign" and moyamoya disease in a child with neurofibromatosis type 1 . Pediatr Neurol. 2017, 70:80. 10.1016/j.pediatrneurol.2017.02.005

27. Vargiami E, Sapountzi E, Samakovitis D, Batzios S, Kyriazi M, Anastasiou A, Zafeiriou DI: Moyamoya syndrome and neurofibromatosis type 1. Ital J Pediatr. 2014, 40:59. 10.1186/1824-7288-40-59

28. Mayl J, Patel H, Chandra T: Moyamoya syndrome in a child with neurofibromatosis type 1: magnetic resonance imaging as a tool for clinical decision making. Cureus. 2017, 9:e1233. 10.7759/cureus.1233

29. Serafini NB, Serafini CB, Vinhas AS, Godinho MB: Moyamoya syndrome associated with neurofibromatosis type 1 in a pediatric patient. An Bras Dermatol. 2017, 92:870-3.

30. Jiménez Caballero PE: [Adult-onset moyamoya disease in a patient with neurofibromatosis type 1]. Neurologia. 2016, 31:139-41. 10.1016/j.nrl.2014.03.006

31. Gold JJ, Dory CE, Levy ML, Crawford JR: Simultaneous moyamoya disease and cervical spinal cord low-grade astrocytoma in a child with neurofibromatosis type 1. BMJ Case Rep. 2013, 2013:bcr2013009812. 10.1136/bcr-2013-009812

32. Darrigo Júnior LG, Valera ET, Machado Ade A, Santos AC, Scrideli CA, Tone LG: Moyamoya syndrome associated with neurofibromatosis type I in a pediatric patient. Sao Paulo Med J. 2011, 129:110-2.

33. Smith M, Heran MK, Connolly MB, et al.: Cerebrovasculopathy in NF1 associated with ocular and scalp defects. Am J Med Genet A. 2011, 155A:380-5. 10.1002/ajmg.a.33788

34. Golomb MR, Smith JL: Poor wound healing after pial synangiosis in 2 children with moyamoya vasculopathy associated with neurofibromatosis type 1. J Child Neurol. 2014, 29:NP101-4. 10.1177/0883073813506611

35. Lai J, Modi L, Ramai D, Tortora M: Tuberous sclerosis complex and polycystic kidney disease contiguous gene syndrome with moyamoya disease. Pathol Res Pract. 2017, 213:410-5. 10.1016/j.prp.2016.12.027

36. de Guzman P, Hamilton M, Khan A, Eesa M, Kirton A: Moyamoya syndrome associated with mucolipidosisII. Can J Neurol Sci. 2014, 41:522-4. 10.1017/s0317167100018618

37. Çevik B, Acu B, Aksoy D, Kurt S: Protein S deficiency and an adult case with moyamoya syndrome that presented with primary intraventricular haemorrhage. Balkan Med J. 2014, 31:180-3. 10.5152/balkanmedj.2014.13028 
38. Klemann C, Kirschner J, Ammann S, et al.: CD59 deficiency presenting as polyneuropathy and moyamoya syndrome with endothelial abnormalities of small brain vessels. Eur J Paediatr Neurol. 2018, 22:870-7. 10.1016/j.ejpn.2018.04.003

39. Cho SM, Di Lorenzo R, Myles JL, Uchino K: Severe hyperhomocysteinemia manifesting as moyamoya vasculopathy and Henoch-Schonlein purpura. Neurology. 2018, 91:321-3. 10.1212/wnl.0000000000005995

40. Ghilain V, Wiame E, Fomekong E, Vincent MF, Dumitriu D, Nassogne MC: Unusual association between lysinuric protein intolerance and moyamoya vasculopathy. Eur J Paediatr Neurol. 2016, 20:777-81. 10.1016/j.ejpn.2016.05.016

41. Zaletel M, Surlan-Popović K, Pretnar-Oblak J, Zvan B: Moyamoya syndrome with arteriovenous dural fistula after head trauma. Acta Clin Croat. 2011, 50:115-20.

42. Dezmalj-Grbelja L, Bosnjak J, Lovrencić-Huzjan A, Ivica M, Demarin V: Moyamoya disease in a patient with brain tumor: case report. Acta Clin Croat. 2010, 49:459-63.

43. Lang M, Moore NZ, Witek AM, Kshettry VR, Bain MD: Microsurgical repair of ruptured aneurysms associated with moyamoya-pattern collateral vessels of the middle cerebral artery: a report of two cases. World Neurosurg. 2017, 105:1042.e5-e10. 10.1016/j.wneu.2017.06.166

44. Choi J, Suthakar P, Farmand F: Fatal outcome in a Hispanic woman with moyamoya syndrome and Graves' disease. Endocrinol Diabetes Metab Case Rep. 2016, 2016: 10.1530/edm-16-0045

45. Sakalli H, Baskin E, Alehan F, Aglldere M, Akova YA, Caner H: Dual anca positivity in a child with moyamoya-like cerebral vascular changes: an unusual presentation with sudden homonymous hemianopsia. Rheumatol Int. 2012, 32:3295-8. 10.1007/s00296-011-2064-y

46. Ahmad AS, Tahir RA, Mitsias PD: Moyamoya vasculopathy with anti-SCL-70 antibodies: a case report and review of the literature. J Clin Neurosci. 2018, 56:177-9. 10.1016/j.jocn.2018.06.055

47. Wegner F, Müller-Ladner U, Meier FM: Reply to 'Moyamoya vasculopathy with anti-SCL-70 antibodies: A case report and review of the literature'. J Clin Neurosci. 2019, 63:281. 10.1016/j.jocn.2019.01.043

48. Troedson C, Wong M, Dalby-Payne J, et al.: Systemic lupus erythematosus due to C1q deficiency with progressive encephalopathy, intracranial calcification and acquired moyamoya cerebral vasculopathy. Lupus. 2013, 22:639-43. 10.1177/0961203313486950

49. Valluri M, Akhondi H, Hyndman M: Moyamoya complicated by thrombotic cerebrovascular accident in a Caucasian woman with collagenous colitis. Neurol Sci. 2018, 39:2007-9. 10.1007/s10072-018-3519-7

50. Wegner F, Mueller-Ladner U, Meier FM: Moyamoya disease and systemic sclerosis (MoSys syndrome): a combination of two rare entities: comment to the authors. Clin Exp Rheumatol. 2016, 34:210.

51. Skeik N, Rumery KK, Udayakumar PD, Crandall BM, Warrington KJ, Sullivan TM: Concurrent Takayasu arteritis with common variable immunodeficiency and moyamoya disease. Ann Vasc Surg. 2013, 27:P240.e13-e18. 10.1016/j.avsg.2012.09.003

52. Hughes JW, Wyckoff JA, Hollander AS, Derdeyn CP, McGill JB: Moyamoya syndrome causing stroke in young women with type 1 diabetes. J Diabetes Complicat. 2016, 30:1640-2. 10.1016/j.jdiacomp.2016.08.004

53. Malik S, Russman AN, Katramados AM, Silver B, Mitsias PD: Moyamoya syndrome associated with Graves' disease: a case report and review of the literature. J Stroke Cerebrovasc Dis. 2011, 20:528-36. 10.1016/j.jstrokecerebrovasdis.2010.03.006

54. Brandicourt P, Bonnet L, Béjot Y, Drouet C, Moulin T, Thines L: Moya-Moya syndrome after cranial radiation for optic glioma with NF1. Case report and literature review of syndromic cases. Neurochirurgie. 2018, 64:63-7. 10.1016/j.neuchi.2017.11.004

55. Reynolds MR, Haydon DH, Caird J, Leonard JR: Radiation-induced moyamoya syndrome after proton beam therapy in the pediatric patient: a case series. Pediatr Neurosurg. 2016, 51:297-301. 10.1159/000446075

56. Manion B, Sung WS: Radiation-induced moyamoya disease after childhood astrocytoma . J Clin Neurosci. 2011, 18:1403-5. 10.1016/j.jocn.2011.01.032

57. Zwagerman NT, Foster K, Jakacki R, Khan FH, Yock TI, Greene S: The development of moyamoya syndrome after proton beam therapy. Pediatr Blood Cancer. 2014, 61:1490-2. 10.1002/pbc.24961

58. Scala M, Vennarini S, Garrè ML, et al.: Radiation-induced moyamoya syndrome after proton therapy in child with clival chordoma: natural history and surgical treatment. World Neurosurg. 2019, 123:306-9. 10.1016/j.wneu.2018.12.048

59. Almeida P, Rocha AL, Alves G, et al.: Moyamoya syndrome after radiation therapy: a clinical report . Eur J Case Rep Intern Med. 2019, 6:001337. 10.12890/2019_001337

60. Guey S, Grangeon L, Brunelle F, et al.: De novo mutations in CBL causing early-onset paediatric moyamoya angiopathy. J Med Genet. 2017, 54:550-7. 10.1136/jmedgenet-2016-104432

61. Bayram AK, Yilmaz E, Per H, et al.: Familial moyamoya disease in two Turkish siblings with same polymorphism in RNF213 gene but different clinical features. Childs Nerv Syst. 2016, 32:569-73. 10.1007/s00381-015-2871-7

62. Luisa SF, Rizzo A, Bedini G, et al.: Microduplication of 15q13.3 and microdeletion of 18q21.32 in a patient with moyamoya syndrome. Int J Mol Sci. 2018, 19:20. 10.3390/ijms19113675

63. Keylock A, Hong Y, Saunders D, et al.: Moyamoya-like cerebrovascular disease in a child with a novel mutation in myosin heavy chain 11. Neurology. 2018, 90:136-8. 10.1212/wnl.0000000000004828

64. Toldo I, Po C, Morao V, et al.: Moyamoya syndrome and 6p chromosome rearrangements: expanding evidences of a new association. Eur J Paediatr Neurol. 2016, 20:766-71. 10.1016/j.ejpn.2016.02.008

65. Melis M, Cau M, Corraine S, Secci S, Addis M, Melis M: Cerebral cavernous malformations and unilateral moyamoya in a patient with a new mutation in the KRIT-1/CCM1 gene. Cerebrovasc Dis. 2014, 38:311-2. 10.1159/000368215

66. Wallace S, Guo DC, Regalado E, et al.: Disrupted nitric oxide signaling due to GUCY1A3 mutations increases risk for moyamoya disease, achalasia and hypertension. Clin Genet. 2016, 90:351-60. 10.1111/cge.12739

67. Schilter KF, Steiner JE, Demos W, et al.: RNF213 variants in a child with PHACE syndrome and moyamoya vasculopathy. Am J Med Genet A. 2017, 173:2557-61. 10.1002/ajmg.a.38258

68. Harel T, Posey JE, Graham BH, Walkiewicz M, Yang Y, Lalani SR, Belmont JW: Atypical presentation of moyamoya disease in an infant with a de novo RNF213 variant. Am J Med Genet A. 2015, 167:2742-7. 10.1002/ajmg.a.37230 
69. Teo M, Johnson JN, Bell-Stephens TE, et al.: Surgical outcomes of Majewski osteodysplastic primordial dwarfism type II with intracranial vascular anomalies. J Neurosurg Pediatr. 2016, 25:717-23. 10.3171/2016.6.Peds 16243

70. Kılıç E, Utine E, Unal S, et al.: Medical management of moyamoya disease and recurrent stroke in an infant with Majewski osteodysplastic primordial dwarfism type II (MOPD II). Eur J Pediatr. 2012, 171:1567-71. 10.1007/s00431-012-1732-6

71. Bang GM, Kirmani S, Patton A, Pulido JS, Brodsky MC: “Ocular moyamoya” syndrome in a patient with features of microcephalic osteodysplastic primordial dwarfism type II. J AAPOS. 2013, 17:100-2. 10.1016/j.jaapos.2012.09.007

72. Prontera P, Rogaia D, Mencarelli A, et al.: Juvenile moyamoya and craniosynostosis in a child with deletion 1p32p31: expanding the clinical spectrum of 1p32p31 deletion syndrome and a review of the literature. Int J Mol Sci. 2017, 18:1998. 10.3390/ijms18091998

73. Vo Van P, Sabouraud P, Mac G, Abely M, Bednarek N: Moyamoya disease associated with hereditary spherocytosis. Pediatr Neurol. 2011, 44:69-71. 10.1016/j.pediatrneurol.2010.08.002

74. Yadegari S, Aminian A: Management in rare association of moyamoya syndrome and hereditary spherocytosis. Minerva Pediatr. 2017, 69:368-9. 10.23736/s0026-4946.16.04427-3

75. Gait-Carr E, Connolly DJ, King D: Moyamoya syndrome associated with hereditary spherocytosis: an emerging clinical entity. J Pediatr Hematol Oncol. 2017, 39:233-4. 10.1097/mph.0000000000000789

76. Karvandian K, Khan ZH, Zebardast J, Miri SR: Laparoscopic splenectomy in a child with moyamoya syndrome, hereditary spherocytosis, and interstitial lung disease: a mere coincidence or partnership based on genetic similarities. Case Rep Anesthesiol. 2011, 2011:253434. 10.1155/2011/253434

77. Ray A, Rodriguez N: Cerebral infarction in the setting of moyamoya in a pediatric patient with sickle $\beta+-$ thalassemia. Pediatr Hematol Oncol. 2011, 28:535-7. 10.3109/08880018.2011.583976

78. Al-Hawsawi ZM, Al-Zaid MA, Barnawi AI, Yassine SM: Fanconi anemia associated with moyamoya disease in Saudi Arabia. Saudi Med J. 2015, 36:233-5. 10.15537/smj.2015.2.9978

79. Soares D, Bullock R, Ali S: Moyamoya syndrome in sickle cell anaemia: a cause of recurrent stroke . BMJ Case Rep. 2014, 2014:bcr2014203727. 10.1136/bcr-2014-203727

80. Janczar S, Fogtman A, Koblowska M, et al.: Novel severe hemophilia A and moyamoya (SHAM) syndrome caused by Xq28 deletions encompassing F8 and BRCC3 genes. Blood. 2014, 123:4002-4. 10.1182/blood-2014$02-553685$

81. Delavari N, Strahle J, Maher CO: Moyamoya syndrome associated with hemoglobin Southampton (Casper) . Pediatr Neurosurg. 2013, 49:307-10. 10.1159/000367973

82. Inati A, Tourjuman O, Bizri D, et al.: A rare case of Moyamoya syndrome in a $\beta$-thalassemia major patient . Blood Cells Mol Dis. 2013, 51:149-50. 10.1016/j.bcmd.2013.05.005

83. Ebert JJ, Sisk RA: CRAO in moyamoya syndrome associated with Southampton hemoglobinopathy . Ophthalmic Surg Lasers Imaging Retina. 2019, 50:e166-70. 10.3928/23258160-20190503-17

84. Lavin M, Jenkins PV, Keenan C, White B, Betts DR, O'Donnell JS, O'Connell NM: X-linked moyamoya syndrome associated with severe haemophilia A. Haemophilia. 2016, 22:51-4. 10.1111/hae.12806

85. Komur M, Unal S, Okuyaz C, Ozgur A: Moyamoya syndrome associated with sickle cell trait in a child . Brain Dev. 2014, 36:545-7. 10.1016/j.braindev.2013.07.010

86. Agrawal R, Berube C, Steinberg G, George TI: Moyamoya syndrome with sickle cell trait. Int J Lab Hematol. 2013, 35:8-9. 10.1111/ijlh.12048

87. El Beltagi AH, El-Sheikh A, El-Saif R, Norbash A: Ivy sign in mildly symptomatic $\beta$-thalassemia intermedia, with development of moyamoya disease. Neuroradiol J. 2014, 27:23-8. 10.15274/nrj-2014-10003

88. Shiari R, Tabatabaei Nodushan SM, Mohebbi MM, Karimzadeh P, Javadzadeh M: Moyamoya syndrome associated with Henoch-Schönlein purpura. Iran J Child Neurol. 2016, 10:71-4.

89. Horev L, Lees MM, Anteby I, Gomori JM, Gunny R, Ben-Neriah Z: Oculoectodermal syndrome with coarctation of the aorta and moyamoya disease: expanding the phenotype to include vascular anomalies. Am J Med Genet A. 2011, 155:577-81. 10.1002/ajmg.a.33104

90. Tavares Bello C, Barreiros C, Gil I, Vasconcelos C: Down syndrome and moyamoya disease: unusual cause of stroke. BMJ Case Rep. 2017, 2017:bcr-2017-219894. 10.1136/bcr-2017-219894

91. Gupta M, Choudhri OA, Feroze AH, Do HM, Grant GA, Steinberg GK: Management of moyamoya syndrome in patients with Noonan syndrome. J Clin Neurosci. 2016, 28:107-11. 10.1016/j.jocn.2015.11.017

92. Cullu N, Karakas E, Karakas O, Deveer M, Calik M, Boyaci FN: Childhood moyamoya disease accompanying Leigh syndrome. J Pak Med Assoc. 2013, 63:1538-40.

93. Rocha R, Soro I, Leitão A, Silva ML, Leão M: Moyamoya vascular pattern in Alagille syndrome. Pediatr Neurol. 2012, 47:125-8. 10.1016/j.pediatrneurol.2012.04.014

94. Manjila S, Miller BR, Rao-Frisch A, Otvos B, Mitchell A, Bambakidis NC, De Georgia MA: Moyamoya disease associated with asymptomatic mosaic Turner syndrome: a rare cause of hemorrhagic stroke. J Stroke Cerebrovasc Dis. 2014, 23:1242-4. 10.1016/j.jstrokecerebrovasdis.2013.07.038

95. Degirmenci E, Bir LS, Yagcı B, Nazliel B, Siva A: Moyamoya syndrome or Behçet's disease?. Int J Rheum Dis. 2014, 17:920-2. 10.1111/1756-185x.12478

96. Fierini F, Barilaro A, Giambene B, Carlucci G, Grandi V, Maio V, Pantoni L: Moyamoya in a patient with Sneddon's syndrome. Clin Neurol Neurosurg. 2015, 129:34-6. 10.1016/j.clineuro.2014.12.001

97. Delaney S, O'Connor G, Reardon W, et al.: Extracranial and intracranial vasculopathy with "moyamoya phenomenon” in association with Alagille syndrome. Front Neurol. 2018, 9:1194. 10.3389/fneur.2018.01194

98. Freeman J, Deleyiannis F, Bernard TJ, Fenton LZ, Somme S, Wilkinson CC: Moyamoya in a patient with Smith-Magenis syndrome. Pediatr Neurosurg. 2017, 52:195-204. 10.1159/000459627

99. Pavanello M, Severino M, D'Antiga L, Castellan L, Calvi A, Colledan M, Gandolfo C: Pretransplant management of basilar artery aneurysm and moyamoya disease in a child with Alagille syndrome. Liver Transpl. 2015, 21:1227-30. 10.1002/lt.24187

100. Vimalesvaran S, Nachiappan N, Sithamparanathan Y: Moyamoya syndrome in a Malaysian child with Down syndrome. J Paediatr Child Health. 2013, 49:865-7. 10.1111/jpc.12239

101. Kaufman T, White A: Moyamoya in a patient with FIRES: a first case report . Neurodiagn J. 2017, 57:17-26. 
10.1080/21646821.2016.1270652

102. Martel C, Robertson R, Williams FB, Moore RC, Clark A: Anesthetic management of a parturient with PHACE syndrome for cesarean delivery. A A Case Rep. 2015, 5:176-8. 10.1213/xaa.0000000000000208

103. Hong CS, Wang AC, Bonow RH, Abecassis IJ, Amlie-Lefond C, Ellenbogen RG: Moyamoya disease in a patient with VACTERL association. World Neurosurg. 2016, 89:729-7. 10.1016/j.wneu.2016.01.059

104. Nezzar H, Mbekeani JN, Dalens H: Morning glory syndrome with carotid and middle cerebral artery casculopathy. Optom Vis Sci. 2015, 92:437-41. 10.1097/opx.0000000000000727

105. Béjot Y, Barnay JL, Chavent A, et al.: Subarachnoid hemorrhage revealing moyamoya syndrome in a patient with May-Hegglin anomaly. Neurologist. 2017, 22:204-5. 10.1097/nrl.0000000000000150

106. Gross BA, Ropper AE, Du R: Association of mesial temporal sclerosis and moyamoya syndrome. Clin Neurol Neurosurg. 2013, 115:106-7. 10.1016/j.clineuro.2012.04.006

107. Lohani S, Madsen JR, Bergin AM, Smith ER: Moyamoya disease with mesial temporal sclerosis . J Neurosurg Pediatr. 2013, 11:713-6. 10.3171/2013.3.Peds12437

108. Diaz UJ, Cabán-Martinez AJ, Halder GE: Presentation with recurrent intractable headache: a patient with moyamoya syndrome: case report. Neurol Med Chir (Tokyo). 2014, 54:133-5. 10.2176/nmc.cr2012-0134

109. Azzam DB, Sharma AN, Tiourin E, Chan AY: A case report of moyamoya disease presenting as headache in a 35-year-old Hispanic man. Cureus. 2019, 11:e4426. 10.7759/cureus.4426

110. Elia M: Bilateral visual field loss in an adolescent girl with migraine headaches . JAMA Ophthalmol. 2018, 136:1072-3. 10.1001/jamaophthalmol.2017.6815

111. Verdure P, Le Moigne O, Massardier EG, Vanhulle C, Tollard E, Maltête D: [Migraine-like headache and ocular malformations may herald moyamoya syndrome]. Rev Neurol (Paris). 2012, 168:460-1. 10.1016/j.neurol.2011.09.011

112. Tozzi E, Antenucci A, Di Loreto S, Maresca M, Farello G, Massimi L: Moyamoya disease and headache: case report. J Headache Pain. 2015, 16:A73. 10.1186/1129-2377-16-s1-a73

113. Pinardi F, Stracciari A, Spinardi L, Guarino M: Postpneumococcal moyamoya syndrome case report and review of the postinfective cases. BMJ Case Rep. 2013, 2013:bcr2012006726. 10.1136/bcr-2012-006726

114. Trombatore P, Lozupone E, Gaudino S, et al.: A rare case of postinfectious moyamoya syndrome: case report and review of the literature. World Neurosurg. 2020, 140:213-8. 10.1016/j.wneu.2020.05.082

115. Sajja A, Tsering D, Mooser AC, DeFreitas TA, Carpenter J, Magge SN: Patient with severe moyamoya disease who presents with acute cortical blindness. Stroke. 2017, 48:126-9. 10.1161/strokeaha.116.015548

116. Papavasileiou E, Sobrin L, Papaliodis GN: Ocular ischemic syndrome presenting as retinal vasculitis in a patient with moyamoya syndrome. Retin Cases Brief Rep. 2015, 9:170-2. 10.1097/icb.0000000000000129

117. Santos AF, Pinho J, Ferreira C, Pereira C, Ribeiro M, Rocha J: "The fainted man:" hypoperfusion encephalopathy in a patient with moyamoya. J Neuropsychiatry Clin Neurosci. 2014, 26:31-3. 10.1176/appi.neuropsych.13020026

118. Born H, Wineland A, Rutter MJ: Neurologically acquired laryngomalacia in a pediatric patient with moyamoya: a case report and literature review. Int J Pediatr Otorhinolaryngol. 2019, 116:34-7. 10.1016/j.ijporl.2018.10.001

119. Lai J, Patel A, Dandurand C, Gooderham P, Lu S: Depression and catatonia: a case of neuropsychiatric complications of moyamoya disease. Cureus. 2018, 10:e3460. 10.7759/cureus.3460

120. Indorewalla KK, McArdle M, Tomlinson E, Piryatinsky I: Neuropsychological profile associated with moyamoya disease: a case report. NeuroRehabilitation. 2020, 46:603-11. 10.3233/nre-192903

121. Richards M, Grzenda A, Nelson E, Gitlin M: Psychiatric comorbidity in moyamoya disease and preliminary guidelines for treatment. Am J Psychiatry. 2019, 176:269-74. 10.1176/appi.ajp.2018.18040404

122. Hamamoto Filho PT, Lira CCS, Zanini MA: Moyamoya syndrome manifesting with choreiform movements. Neuropediatrics. 2018, 49:80-1. 10.1055/s-0037-1607967

123. Platzen J, Berlit P, Kraemer M: Chorea gravidarum associated with moyamoya angiopathy treated with alpha-methyldopa. Clin Neurol Neurosurg. 2017, 158:126. 10.1016/j.clineuro.2017.04.023

124. Laatar F, Kacem I, Nasri A, Ben Djebara M, Gargouri A, Gouider R: Generalized dystonia: unusual mode of revelation of moyamoya disease. Tunis Med. 2017, 95:504-5.

125. Greene S, Bansal L, Coffman KA, Nardone R, Zuccoli G: Pial synangiosis ameliorates movement disorders in the absence of prior stroke in moyamoya disease. J Child Neurol. 2016, 31:646-51. 10.1177/0883073815609152

126. Tam HH, Amiras D, Patel M, Win Z: An unusual cause of hemiplegia in a 28-year-old woman . Br J Hosp Med (Lond). 2013, 74:526-7. 10.12968/hmed.2013.74.9.526

127. Pandey P, Bell-Stephens T, Steinberg GK: Patients with moyamoya disease presenting with movement disorder. J Neurosurg Pediatr. 2010, 6:559-66. 10.3171/2010.9.Peds10192

128. Lamônica DA, Ribeiro CD, Ferraz PM, Tabaquim ML: [Moyamoya disease: impact on the performance of oral and written language]. Codas. 2016, 28:661-5. 10.1590/2317-1782/20162016010

129. Zalonis I, Christidi F, Kararizou E, Triantafyllou NI, Spengos K, Vassilopoulos D: Cognitive deficits presenting as psychiatric symptoms in a patient with moyamoya disease. Psychol Rep. 2010, 107:727-32. 10.2466/02.13.15.22.Pr0.107.6.727-732

130. Wu TY, Smith A, Kilfoyle D: Hemifacial spasm leading to diagnosis of moyamoya disease . N Z Med J. 2013, 126:92-5.

131. Bidaki R, Zarepur E: Intermittent hemiplegia in a boy with primary moyamoya disease: a case report from Iran. Iran J Child Neurol. 2017, 11:65-8.

132. Gül F, Berçin S, Müderris T, Yalçıner G, Ünal Ö, Kırış M: Unilateral sudden hearing loss: a rare symptom of moyamoya disease. Kulak Burun Bogaz Ihtis Derg. 2016, 26:114-7.

133. Schranz D, Kerst G, Menges T, et al.: Transcatheter creation of a reverse Potts shunt in a patient with severe pulmonary arterial hypertension associated with moyamoya syndrome. Eurointervention. 2015, 11:121. 10.4244/EIJV11I1A21

134. Kızılkaya MH, Uysal F, Gürbüz E, Taşkapılıŏlu M, Bostan ÖM: Atypical presentation of moyamoya disease with pulmonary hypertension: a case report. Anatol J Cardiol. 2018, 19:350-1.

10.14744/AnatoljCardiol.2018.65642 
135. Migliore F, Iorember FM, Gedalia A: 6-year-old with severe hypertension. Clin Pediatr (Phila). 2018, 57:2414. 10.1177/0009922817692315

136. Witmer MT, Levy R, Yohay K, Kiss S: Ophthalmic artery ischemic syndrome associated with neurofibromatosis and moyamoya syndrome. JAMA Ophthalmol. 2013, 131:538-9. 10.1001/jamaophthalmol.2013.2902

137. Savio K, Mittino D, Terazzi E, Divenuto I, Fossaceca R, Carriero A, Monaco F: Moyamoya disease and arteriovenous fistula of the epiaortic vessels. Neurol Sci. 2010, 31:821-4. 10.1007/s10072-010-0331-4

138. Karsten MB, Oliveira C, Segal AZ, Scott RM: Central retinal artery occlusion occurring 30 years after successful revascularization surgery for moyamoya disease: case report. Acta Neurochir (Wien). 2020, 162:2589-92. 10.1007/s00701-020-04501-8

139. Katsman D, Klufas MA, Sarraf D, Sadda S: Retinal arterial tortuosity in moyamoya disease . JAMA Ophthalmol. 2016, 134:111-4. 10.1001/jamaophthalmol.2015.4645

140. Güçlü H, Gurlu VP, Ozal SA, Esgin H: A moyamoya patient with bilateral consecutive branch retinal vein occlusion. Neuroophthalmology. 2016, 40:93-6. 10.3109/01658107.2016.1148174

141. Jiang T, Perry A, Dacey RG, Jr., Zipfel GJ, Derdeyn CP: Intracranial atherosclerotic disease associated with moyamoya collateral formation: histopathological findings. J Neurosurg. 2013, 118:1030-4. 10.3171/2013.1.Jns12565

142. Toscano M, Puledda F, Viganò A, Vicenzini E, Guidetti G, Lenzi GL, Di Piero V: Hemodynamic features of non-aneurysmal subarachnoid hemorrhage in a case of familial moyamoya disease: a transcranial Doppler ultrasound study. Eur Neurol. 2014, 72:330-2. 10.1159/000364862

143. Beyaz P, Khan N, Baltsavias G: Multiple anomalies in the origin and course of vertebral arteries and aberrant right subclavian artery in a child with moyamoya syndrome. BMJ Case Rep. 2018, 2018:bcr-2017013464. 10.1136/bcr-2017-013464

144. Lutz T, Mönnings P, Ayzenberg I, Lukas C: Twig-like middle cerebral artery: a seldom vessel anomaly of important relevance. Clin Neuroradiol. 2018, 28:441-3. 10.1007/s00062-017-0613-9

145. Reid AJ, Bhattacharjee MB, Regalado ES, Milewicz AL, El-Hakam LM, Dauser RC, Milewicz DM: Diffuse and uncontrolled vascular smooth muscle cell proliferation in rapidly progressing pediatric moyamoya disease. J Neurosurg Pediatr. 2010, 6:244-9. 10.3171/2010.5.Peds09505

146. Garoon R, Carvounis PE: Central retinal vein occlusion with bilateral stenosis of the internal carotid arteries. Lancet. 2015, 385:914. 10.1016/s0140-6736(15)60124-6

147. Moceri P, Laïk J, Bouvaist H, Fraisse A, Ferrari E: Peripheral pulmonary artery stenoses in the setting of moyamoya. Eur Heart J Cardiovasc Imaging. 2016, 17:575. 10.1093/ehjci/jew006

148. Rajanala AP, Le HT, Gill MK: Central retinal artery occlusion as initial presentation of moyamoya disease in a middle-aged woman. Am J Ophthalmol Case Rep. 2020, 18:100705. 10.1016/j.ajoc.2020.100705

149. Koduri S, Wilkinson DA, Griauzde JM, Gemmete JJ, Maher CO: Development of bilateral dural arteriovenous fistulae following pial synangiosis for moyamoya syndrome: case report. J Neurosurg Pediatr. 2019, 24:9-13. 10.3171/2019.2.Peds18603

150. Livesay J, Johnson J: ST-elevation myocardial infarction (STEMI) in a patient with moyamoya disease . Case Rep Cardiol. 2019, 2019:7124072. 10.1155/2019/7124072

151. Kraemer M, Diehl RR, Diesner F, Berlit P, Khan N: Differential diagnosis between cerebral ischemia, focal seizures and limb shaking TIAs in moyamoya disease. Br J Neurosurg. 2012, 26:896-8. 10.3109/02688697.2012.697213

152. Ozer M, Merchant K, Manning Z, Goksu SY, Juneja K, Fennell VS: Postpartum-onset moyamoya disease: a rare cause of stroke in unexpected. Case Rep Neurol Med. 2020, 2020:7689450. 10.1155/2020/7689450

153. Chaughtai S, Chaughtai Z, Haider MS, et al.: Subacute stroke in a young female: a case of moyamoya syndrome initially anchoring with anxiety. Case Rep Med. 2019, 2019:7919568. 10.1155/2019/7919568

154. Ilyayeva E, Nada K, Farahi Far R, Albright K, Gujral MK, Gold M: Bilateral cerebrovascular stroke as an initial presenting symptom of moyamoya disease. Case Rep Crit Care. 2018, 2018:2591494. 10.1155/2018/2591494

155. Mohammadi O, Krieger D, Butt I, Danckers M: A case of delayed diagnosis of moyamoya disease after recurrent strokes. Cureus. 2019, 11:e6446. 10.7759/cureus.6446

156. Omer S, Zbyszynska R, Kirthivasan R: Peek through the smoke: a report of moyamoya disease in a 32-yearold female patient presenting with ischaemic stroke. BMJ Case Rep. 2018, 2018:bcr-2017-221685. 10.1136/bcr-2017-221685

157. Casserly CS, Salmon A, Ramsay DA, Pelz DM, Lownie SP, Strong MJ: Multiple cerebral infarcts in patient with moyamoya disease. Can J Neurol Sci. 2012, 39:378-82. 10.1017/s0317167100013548

158. Djedovic G, Verstappen R, Matiasek J, Engelhardt TO, Pierer G, Rieger UM: Occult moyamoya disease causing fulminant infarction after septorhinoplasty. Case Rep Plast Surg Hand Surg. 2014, 1:26-8. 10.3109/23320885.2014.973882

159. Bechan RS, van Rooij WJ: Endovascular treatment of a ruptured flow aneurysm of the heubner artery as part of a moyamoya collateral network in a young patient with an occluded middle cerebral artery. Interv Neuroradiol. 2014, 20:791-5. 10.15274/inr-2014-10071

160. Heijmen L, van Dijk EJ, Goraj B, van Laarhoven HW: Moyamoya disease misdiagnosed as leptomeningeal metastases. J Clin Oncol. 2012, 30:382-5. 10.1200/jco.2012.43.3227

161. Mélot A, Chazot JV, Troude L, De la Rosa S, Brunel H, Roche PH: [Ruptured posterior ethmoidal artery aneurysm and Moyamoya disease in an adult patient. Case report]. Neurochirurgie. 2016, 62:171-3. 10.1016/j.neuchi.2016.04.001

162. Harreld JH, Zomorodi AR: Embolization of an unruptured distal lenticulostriate aneurysm associated with moyamoya disease. Am J Neuroradiol. 2011, 32:E42-3. 10.3174/ajnr.A1993

163. Abuoliat ZA, AlFarhan BA, Alshahrani AA, AlFarhan AA, Almuntashri MA, Alotaibi N: Atypical location of intracerebral hemorrhage in moyamoya disease. Cureus. 2017, 9:e1948. 10.7759/cureus.1948

164. Alcalá-Cerra GA, Moscote-Salazar LR, Barrios RS, Niño-Hernández LM, Gutiérrez Paternina JJ: Nonaneurysmal subarachnoid hemorrhage as presentation of moyamoya disease in an adult. Surg Neurol Int. 2011, 2:80.

165. Noureldine MH, Saikali I, Nassif A, Chahinian R, Sweid A, Kikano R, Mawad M: Pediatric moyamoya 
presenting as a subarachnoid hemorrhage from a ruptured anterior cerebral artery aneurysm. World Neurosurg. 2020, 134:123-7. 10.1016/j.wneu.2019.10.157

166. Donohue MM, Moore A, Shibata D, Ebel-Caswell S, Becker KJ: Transcranial Doppler ultrasound CO2 challenge complicated by subarachnoid hemorrhage in patient with moyamoya syndrome. Neurocrit Care. 2010, 13:243-6. 10.1007/s12028-009-9314-9

167. Varanasi LC, Brown J, Athayde N: Postpartum seizure and subarachnoid haemorrhage secondary to moyamoya disease. Case Rep Obstet Gynecol. 2019, 2019:6132835. 10.1155/2019/6132835

168. Plans Galván O, Manciño Contreras JM, Coy Serrano A, Campos Gómez A, Toboso Casado JM, Ricart Martí P: [Intraparenchymal haemorrhage secondary to moyamoya disease in a white patient] . Neurologia (Engl Ed). 2019, 34:553-5. 10.1016/j.nrl.2016.12.002

169. Schödel P, Brawanski A, Friedrich M, Schlachetzki F, Heiss P, Schebesch KM: Fatal hemorrhagic stroke in a Caucasian girl with moyamoya disease. Childs Nerv Syst. 2013, 29:1381-5. 10.1007/s00381-013-2089-5

170. Ko BL, Unkel JH: Dental management of a pediatric patient with moyamoya syndrome: a rare clinical entity . Pediatr Dent. 2018, 40:56-8.

171. Bo H, Avenetti D, Kratunova E: Dental management considerations in a pediatric patient with moyamoya disease. J Dent Child (Chic). 2017, 84:100-5.

172. Kraemer M, Lee SI, Ayzenberg I, et al.: Headache in Caucasian patients with moyamoya angiopathy - a systematic cohort study. Cephalalgia. 2017, 37:496-500. 10.1177/0333102416643516

173. Klasen H, Britton J, Newman M: Moyamoya disease in a 12-year-old Caucasian boy presenting with acute transient psychosis. Eur Child Adolesc Psychiatry. 1999, 8:149-53. 10.1007/s007870050096

174. Kraemer M, Trakolis L, Platzen J, et al.: Movement symptoms in European moyamoya angiopathy - first systematic questionnaire study. Clin Neurol Neurosurg. 2017, 152:52-6. 10.1016/j.clineuro.2016.11.017

175. Scott RM, Smith JL, Robertson RL, Madsen JR, Soriano SG, Rockoff MA: Long-term outcome in children with moyamoya syndrome after cranial revascularization by pial synangiosis. J Neurosurg. 2004, 100:142-9. 10.3171/ped.2004.100.2.0142

176. Setzen G, Cacace AT, Eames F, et al.: Central deafness in a young child with Moyamoya disease: paternal linkage in a Caucasian family: two case reports and a review of the literature. Int J Pediatr Otorhinolaryngol. 1999, 48:53-76. 10.1016/s0165-5876(99)00004-X

177. Arias EJ, Derdeyn CP, Dacey RG, Jr., Zipfel GJ: Advances and surgical considerations in the treatment of moyamoya disease. Neurosurgery. 2014, 74:116-25. 10.1227/neu.0000000000000229

178. Stejskal V, Šteiner I, Hornychová H, Krůpa P, Kanta M: Moyamoya disease associated with fibromuscular dysplasia of intrapulmonary bronchial arteries-a case report. Cardiovasc Pathol. 2020, 45:107182. 10.1016/j.carpath.2019.107182

179. Jansen JN, Donker AJ, Luth WJ, Smit LM: Moyamoya disease associated with renovascular hypertension . Neuropediatrics. 1990, 21:44-7. 10.1055/s-2008-1071457

180. Kim SJ, Heo KG, Shin HY, et al.: Association of thyroid autoantibodies with moyamoya-type cerebrovascular disease: a prospective study. Stroke. 2010, 41:173-6. 10.1161/strokeaha.109.562264

181. El Ramahi KM, Al Rayes HM: Systemic lupus erythematosus associated with moyamoya syndrome. Lupus. 2000, 9:632-6. 10.1191/096120300678828686

182. Wang CY, Grupke SL, Roberts J, Lee J, Fraser JF: Factors associated with moyamoya syndrome in a Kentucky regional population. J Stroke Cerebrovasc Dis. 2018, 27:793-800. 10.1016/j.jstrokecerebrovasdis.2017.10.016

183. Hall JG, Flora C, Scott CI, Jr., Pauli RM, Tanaka KI: Majewski osteodysplastic primordial dwarfism type II (MOPD II): natural history and clinical findings. Am J Med Genet A. 2004, 130:55-72. 10.1002/ajmg.a.30203

184. Kronenburg A, Braun KP, van der Zwan A, Klijn CJ: Recent advances in moyamoya disease: pathophysiology and treatment. Curr Neurol Neurosci Rep. 2014, 14:423. 10.1007/s11910-013-0423-7

185. Brancati F, Castori M, Mingarelli R, Dallapiccola B: Majewski osteodysplastic primordial dwarfism type II (MOPD II) complicated by stroke: clinical report and review of cerebral vascular anomalies. Am J Med Genet A. 2005, 139:212-5. 10.1002/ajmg.a.31009

186. Bober MB, Khan N, Kaplan J, Lewis K, Feinstein JA, Scott CI Jr., Steinberg GK: Majewski osteodysplastic primordial dwarfism type II (MOPD II): expanding the vascular phenotype. Am J Med Genet A. 2010, 152:960-5. 10.1002/ajmg.a.33252

187. Rahme R, Crevier L, Dubois J, Mercier C: Moyamoya-like vasculopathy and Seckel syndrome: just a coincidence?. Childs Nerv Syst. 2010, 26:983-6. 10.1007/s00381-010-1142-x

188. Gunesli A, Andic C, Alkan O, Erol I, Suner HI: Endovascular treatment of a patient with moyamoya disease and Seckel syndrome: a case report. J Pediatr Neurosci. 2018, 13:245-8. 10.4103/jpn.JPN_96_17

189. Stowe RC, Jimenez-Gomez A, Balasa A, Clark GD: Cockayne syndrome complicated by moyamoya vasculopathy and stroke. Pediatr Neurol. 2018, 86:73-4. 10.1016/j.pediatrneurol.2018.05.002

190. Coughlin DJ, Miller CA, Schuette AJ: Treatment of moyamoya disease and unruptured intracranial aneurysm in floating-harbor syndrome. World Neurosurg. 2017, 104:1049.e1-e6. 10.1016/j.wneu.2017.05.083

191. Sunder TR: Moyamoya disease in a patient with type I glycogenosis . Arch Neurol. 1981, 38:251-3. 10.1001/archneur.1981.00510040077014

192. Baird LC, Smith ER, Ichord R, et al.: Moyamoya syndrome associated with Alagille syndrome: outcome after surgical revascularization. J Pediatr. 2015, 166:470-3. 10.1016/j.jpeds.2014.10.067

193. Spengos K, Kosmaidou-Aravidou Z, Tsivgoulis G, Vassilopoulou S, Grigori-Kostaraki P, Zis V: Moyamoya syndrome in a Caucasian woman with Turner's syndrome. Eur J Neurol. 2006, 13:7-8. 10.1111/j.14681331.2006.01417.x

194. Barrit S: [An Aicardi-Goutières syndrome associated with a quasi-Moyamoya by a biallelic mutation in SAMHD1]. Rev Med Brux. 2018, 39:155-60. 10.30637/2018.16-030

195. Sequeira W, Naseem M, Bouffard DA: An association with birth control pills. Moyamoya. IMJ Ill Med J. 1984, 166:434-6.

196. Peerless SJ: Risk factors of moyamoya disease in Canada and the USA . Clin Neurol Neurosurg. 1997, 99:45-8. 10.1016/s0303-8467(97)00039-5

197. Uchino K, Johnston SC, Becker KJ, Tirschwell DL: Moyamoya disease in Washington State and California . 
Neurology. 2005, 65:956-8. 10.1212/01.wnl.0000176066.33797.82

198. Merkel KH, Ginsberg PL, Parker JC, Jr., Post MJ: Cerebrovascular disease in sickle cell anemia: a clinical, pathological and radiological correlation. Stroke. 1978, 9:45-52. 10.1161/01.str.9.1.45

199. Newman S, Boulter JH, Malcolm JG, Pradilla I, Pradilla G: Outcomes in patients with moyamoya syndrome and sickle cell disease: a systematic review. World Neurosurg. 2020, 135:165-70.

10.1016/j.wneu.2019.11.137

200. Cheng ZJ, Shen YY, Warsame IM, Dai TM, Tu JL: Moyamoya syndrome caused by paroxysmal nocturnal hemoglobinuria. Chin Med J (Engl). 2018, 131:2874-6.

201. Marden FA, Putman CM, Grant JM, Greenberg J: Moyamoya disease associated with hemoglobin Fairfax and beta-thalassemia. Pediatr Neurol. 2008, 38:130-2. 10.1016/j.pediatrneurol.2007.09.011

202. Parker TM, Ward LM, Johnston DL, Ventureya E, Klaassen RJ: A case of moyamoya syndrome and hemoglobin E/beta-thalassemia. Pediatr Blood Cancer. 2009, 52:422-4. 10.1002/pbc.21850

203. Xu F, Tang H, Xiong J, Liu X: Moyamoya disease associated with tuberculum sellae meningioma and cavernous sinus hemangioma. World Neurosurg. 2018, 109:89-95. 10.1016/j.wneu.2017.09.116

204. Morgello S, Laufer H: Quaternary neurosyphilis in a Haitian man with human immunodeficiency virus infection. Hum Pathol. 1989, 20:808-11. 10.1016/0046-8177(89)90078-6

205. Palacio S, Hart RG, Vollmer DG, Kagan-Hallet K: Late-developing cerebral arteropathy after pyogenic meningitis. Arch Neurol. 2003, 60:431-3. 10.1001/archneur.60.3.431

206. Hammond CK, Shapson-Coe A, Govender R, et al.: Moyamoya syndrome in South African children with HIV1 infection. J Child Neurol. 2016, 31:1010-7. 10.1177/0883073816635747

207. Sharfstein SR, Ahmed S, Islam MQ, Najjar MI, Ratushny V: Case of moyamoya disease in a patient with advanced acquired immunodeficiency syndrome. J Stroke Cerebrovasc Dis. 2007, 16:268-72. 10.1016/j.jstrokecerebrovasdis.2007.07.001

208. Desai SS, Paulino AC, Mai WY, Teh BS: Radiation-induced moyamoya syndrome. Int J Radiat Oncol Biol Phys. 2006, 65:1222-7. 10.1016/j.ijrobp.2006.01.038

209. Servo A, Puranen M: Moyamoya syndrome as a complication of radiation therapy. Case report . J Neurosurg. 1978, 48:1026-9. 10.3171/jns.1978.48.6.1026

210. Kestle JR, Hoffman HJ, Mock AR: Moyamoya phenomenon after radiation for optic glioma . J Neurosurg. 1993, 79:32-5. 10.3171/jns.1993.79.1.0032

211. Fukushima Y, Kondo Y, Kuroki Y, Miyake S, Iwamoto H, Sekido K, Yamaguchi K: Are Down syndrome patients predisposed to moyamoya disease?. Eur J Pediatr. 1986, 144:516-7. 10.1007/bf00441756

212. Fukuyama Y, Osawa M, Kanai N: Moyamoya disease (syndrome) and the Down syndrome. Brain Dev. 1992, 14:254-6. 10.1016/s0387-7604(12)80242-7

213. See AP, Ropper AE, Underberg DL, Robertson RL, Scott RM, Smith ER: Down syndrome and moyamoya: clinical presentation and surgical management. J Neurosurg Pediatr. 2015, 16:58-63. 10.3171/2014.12.Peds14563

214. Rison RA: Fluctuating hemiparesis secondary to moyamoya phenomenon in a child with Down syndrome: a case report. Cases J. 2008, 1:240. 10.1186/1757-1626-1-240

215. Parker SE, Mai CT, Canfield MA, et al.: Updated national birth prevalence estimates for selected birth defects in the United States, 2004-2006. Birth Defects Res A Clin Mol Teratol. 2010, 88:1008-16. 10.1002/bdra. 20735 